\title{
Les Amériques chantées et dansées: Panorama des spectacles européens consacrés au Nouveau Monde, du XVle à nos jours
}

\author{
The Americas sung and danced:
}

Panorama of European performances dedicated to the New World, from the 16th century to the present day

\author{
Dr. Pascal Mongne ${ }^{1}$ \\ ${ }^{1}$ Historien de l'art, américaniste. Chargé d'enseignement à l'École du Louvre (Arts des Amériques). Chercheur \\ associé à l'UMR 8096 - Archéologie des Amériques (ArchAm, CNRS - Université Paris 1 Panthéon-Sorbonne)
}

RÉSUMÉ. Depuis cinq siècles, les Amériques ont été vues, décrites, imaginées, interprétées à l'aune des pôles d'intérêts tant politiques qu'intellectuels qui ont animé la Vieille Europe. Ces représentations proviennent des multiples témoignages venus du continent (récits de conquêtes et d'explorations), d'images composées sur place ou recréées en ateliers, et bien entendu d'objets rapportés par milliers. Mais elles sont également issues des exemples produits sur le sol européen : essais philosophiques et romans d'aventures, sculptures et peintures, éléments d'architecture, illustrations populaires et plus tard photographie, cinéma et publicité. Dans ce vaste éventail, un domaine retient notre attention, celui des spectacles chantés et dansés, des mascarades et bals, des défilés et carrousels, des intermèdes et " entrées " d'opéras-royaux ou d'opéras-ballets, des ballets-pantomime et opéras de la période romantique, jusqu'aux opérettes plus récentes. En effet, la tradition du spectacle musical de la vieille Europe a accordé - dès le XVle siècle - une place aux Amériques. Dans le cadre de ce travail, ont pu être recensés 420 spectacles musicaux et dansés ( $\mathrm{y}$ compris les défilés et bals) apparus en Europe, ayant pour thème les Amériques, leurs paysages et leurs habitants ou dans lesquelles ces derniers jouent un rôle même mineur. Ce texte en présente l'évolution au sein d'un panorama historique, première étape d'une étude à venir sur l'iconographie des décors, costumes, machineries et accessoires « américanistes".

ABSTRACT. For five centuries, the Americas have been seen, described, imagined and interpreted according to the political and intellectual interests that animated Old Europe. These representations are based on the multiple testimonies that came from the continent (accounts of conquests and explorations), on images composed on the spot or recreated in workshops, and of course on objects brought back by the thousands. But they were also inspired by examples produced in Europe: philosophical essays and adventure novels, sculptures and paintings, architectural elements, popular illustrations and later photography, cinema and advertising. In this vast range, one domain holds our attention, that of sung and danced performances, masquerades and balls, parades and carousels, interludes and "entrances" of royal operas or ballroom operas, pantomime ballets and operas of the Romantic period, up to the more recent operettas. In fact, the tradition of musical spectacle in old Europe has given - since the 16th century - a place to the Americas. Within the framework of this work, have been identified 420 musical and dance performances (including parades and balls) that were created in Europe, on the theme of the Americas, their landscapes and their inhabitants, or in which the latter play a role, even a minor one. This text presents the evolution of these performances within a historical panorama, the first step of a future study on the iconography of "Americanist" sets, costumes, machinery and accessories.

MOTS-CLÉS. Amériques, art lyrique, danse, opéra, représentations européennes, spectacles.

KEYWORDS. Americas, dance, European representations, lyrical arts, opera, performances.

Par son éloignement, par sa géographie, par la nature et le passé des hommes qui l'ont peuplé, le monde américain n'a cessé d'occuper dans l'histoire des mentalités de l'Ancien monde une place bien particulière, depuis maintenant cinq siècles.

Ainsi, depuis la Découverte, les Amériques ont été vues, décrites, imaginées, interprétées à l'aune des pôles d'intérêts tant politiques qu'intellectuels qui ont orienté la Vieille Europe. Attirant les aventuriers, les conquérants et les élites, inspirant les artistes et les lettrés, fascinant les religieux, les 
philosophes et les hommes de science, touchant même un public populaire friand de sauvagerie, de découvertes spectaculaires et de catastrophes finales, le Nouveau monde a donc été vêtu, masqué de multiples façons d'une image mouvante et sans cesse renouvelée.

Cette image a une histoire. Elle est le reflet du regard porté par l'Europe au cours des temps, lentement élaboré des deux côtés de l'Atlantique, et dont les causes furent multiples et variées : fantasmes et mythes créés par l'inconnu ou l'incompris, découvertes géographiques, attrait des richesses et de l'invraisemblable, conquêtes coloniales, etc. Tous, quels que soient leur nature et leur source, ont créé les représentations d'un Autre et d'un Ailleurs évoluant avec le temps et les connaissances.

Ces représentations proviennent des multiples témoignages venus du continent: récits de conquêtes, de voyages et d'explorations (authentiques ou inventés) ; d'images composées sur place ou recréées en ateliers (depuis les premières gravures jusqu'à la révolution photographique); d'objets rapportés en nombre, œuvres « d'art» ou simples témoins de la vie quotidienne du monde amérindien. Mais elles sont également issues des exemples produits sur le sol européen : essais philosophiques et romans d'aventures, sculptures et peintures, éléments d'architecture, illustrations populaires et plus tard photographie, cinéma et publicité.

Dans ce vaste éventail, un domaine retient notre attention, celui des spectacles chantés et dansés, des mascarades et bals, des défilés et carrousels, des intermèdes et «entrées » d'opéras-royaux ou d'opéras-ballets, des ballets-pantomime et opéras de la période romantique, jusqu'aux opérettes plus récentes. En effet, la tradition du spectacle musical de la vieille Europe, mêlant indissociablement des genres aujourd'hui bien sectorisés, a accordé - dès le XVIe siècle - une place aux Amériques.

Ce texte en présente les principaux aspects et l'évolution au sein d'un panorama historique ${ }^{1}$.

\section{La définition malaisée d'un inventaire}

Le travail de recherche et d'inventaire entrepris depuis une vingtaine de mois et les résultats certainement non définitifs - que nous présentons ici doivent être explicités quant à deux exclusions majeures :

- D'une part les oeuvres littéraires et philosophiques ainsi que les chroniques de voyages : si nombre d'entre elles ont abordé des thèmes américanistes, elles ne furent que très rarement illustrées et par nature ne sont pas des spectacles (bien qu' elles en fussent souvent la source).

- D'autre part le théâtre (comédie, tragédie, drame, etc.) : cette forme de spectacle stricto sensu faisant bien souvent appel à des décors et des costumes aurait pu en revanche trouver sa place dans cet inventaire. En effet, nombreuses furent les pièces de théâtre ayant pour sujet (principal ou mineur) les Amériques. Cependant ces œuvres, proches du monde littéraire et dont les cahiers sont en fait peu illustrés, sont éloignées de notre domaine et pour cette raison ne sont pas abordées ici. Nous renvoyons aux recherches menées à ce titre et notamment au travaux pionniers de Chinard (1913).

Sachons que l'inventaire que nous avons constitué est fondé sur le travail incontournable de Michael Pisani ${ }^{2}$ qui, dans Imagining Native America in Music (2005, voir bibliographie), présente une étude détaillée de l'évolution des spectacles musicaux, lyriques et chorégraphiques consacrés aux Amérindiens. Un imposant listier en est issu, non publié pour des raisons techniques mais

\footnotetext{
${ }^{1}$ Le présent document n'est pas une étude musicologique que l'auteur n'aurait pu conduire mais un inventaire d'histoire de l'art s'inscrivant dans l'axe des travaux qu'il mène depuis plusieurs années sur l'image des Amériques développée par la Vieille Europe depuis cinq siècles (à ce titre voir Mongne, 2016, 2018a, 2018b, 2019).

${ }^{2}$ Michael Pisani est décédé en juillet 2019. 
consultable sur la Toile : A Chronological Listing of Musical Works on American indian Subjects Composed since 1608 (2006, voir bibliographie). Cependant rassemblant quelque 1200 entrées, cet inventaire n'est consacré qu'au monde indigène, excluant généralement les œuvres consacrées à l'Amérique coloniale ou contemporaine. Il inclut de plus les pièces instrumentales (symphonies, chants, etc.) ainsi que les œuvres créées aux Etats-Unis : les unes comme les autres sortant de notre domaine. Ce travail réalisé il y a maintenant plus de douze ans et dans lequel nous avons pu compter plus de 140 spectacles européens, est inévitablement incomplet.

Un nouvel inventaire a donc été entrepris par nos soins, fondé bien entendu sur celui de Pisani ainsi que sur le lent dépouillement des ouvrages publiés, des livrets et des archives accessibles, incluant la recherche patiente et prudente des informations publiées sur la Toile par des institutions et organismes spécialisés, voire par des particuliers. En l'absence des livrets (ou de résumés) dont le contenu nous informent clairement de la nature de l'intrigue, la sélection de certaines œuvres a été fondée sur les titres fournissant des indications géographiques, historiques ou onomastiques précises (Voir Diagramme et Listier).

À ce jour, ont pu être recensés 420 spectacles musicaux et dansés (y compris les défilés et bals) apparus en Europe, ayant pour thème les Amériques, leurs paysages et leurs habitants ou dans lesquelles ces derniers jouent un rôle même mineur (y compris dans les intrigues se déroulant en Europe). Dans cet ensemble imposant, les créations italiennes et françaises sont de loin les plus nombreuses (respectivement 133 et 132 opus), suivies par les oeuvres germaniques (72), anglaises (42) et espagnoles (22). Les 19 réalisations restantes sont réparties entre la Russie (3), le Portugal (3), la République Tchèque (3), la Belgique (2), la Hollande (2), la Pologne (1), le Danemark (1), la Hongrie (1), la Slovaquie (1), la Suède (1) et la Suisse (1). ${ }^{3}$

\section{Les Amériques imaginées et vêtues : évolution d'un genre}

Construction lente et complexe d'un imaginaire européen géocentré, l'évolution des spectacles consacrés aux Amériques peut être ordonnée sur quatre phases chronologiques, chacune présentant ses propres caractéristiques, reflets des connaissances, des a priori culturels et des goûts des élites.

\section{Première époque (XVle siècle) :}

\section{L’Amérique découverte et interprétée}

Ce premier siècle de contacts entre le Nouveau monde et l'Ancien ne semble avoir livré que peu de spectacles dans lesquels l'Amérique joua ne serait-ce qu'un rôle mineur, alors qu'à la même époque les objets provenant des entradas et des conquêtes (Antilles, côtes du Brésil, Mexique, Floride et Texas, Amérique centrale, Pérou, etc.) arrivaient en très grand nombre dans les cabinets de Curiosités et défrayaient les chroniques par leurs techniques (plumasserie, orfèvrerie) et surtout leur étrangeté ${ }^{4}$.

Une explication à cette rareté pourrait résider dans la difficulté d'identification des deux Indes, encore souvent confondues tant dans les récits de voyages que dans les témoins matériels.

\footnotetext{
${ }^{3}$ Le choix de l'origine d'une œuvre a été fondé sur plusieurs caractères généralement associés : langue du titre et du livret, nationalité du compositeur et du librettiste, enfin lieu de création. Si l'identification fut aisée pour la très grande majorité des entrées, en revanche, la question a pu être posée pour plusieurs œuvres, comme /l pomo d'Oro (des Italiens Cesti et Sbarra, créée à la cour de Vienne en 1668) ou bien // Viaggatore americano (de von Dittersdorft, créée à Johannesberg en 1771 pour le PrinceEvêque de Breslau) et que nous considérons donc comme " germaniques ". À l'instar de ces deux opéras - joués certes en italien - nous avons cependant considéré que le lieu de création, reflétant les goûts d'un public local et d'une élite commanditaire, devaient primer.

${ }^{4}$ Voir Honour, 1975. 
Cette confusion perdurera jusque milieu du XVIIe siècle puisqu'il nous est possible de noter une dizaine de ballets à entrées dont les titres, pourtant évocateurs (Grand ballet des Etrangers de 1598, Ballet des Indiens de 1608, Ballet des Indiens de 1621, Ballet des Princes indiens de 1634), font en fait plutôt référence aux Indes orientales (Moureau, 2004, passim).

En fait, entre sept et neuf spectacles mettant en scène des Américains peuvent être comptés durant cette première époque. Le plus ancien pourrait avoir été Der Triumph Kaiser Maximilians si celui-ci avait été représenté, ce qui ne fut probablement jamais le cas faute de moyens financiers (Feest, 2014, p. 295-297). De nombreuses gravures préparatoires furent cependant réalisées par de grands noms (Altdorfer,1513-15 ; Burgkmair, 1516-18) signalant l'importance du projet imaginé dès 1512 et qui devait, au cours d'un défilé à la gloire de l'empereur du Saint-Empire romain germanique, montrer les peuples de différentes parties du monde. Parmi ceux-là, et bien que sous l'appellation « Peuple de Calicut » (région du Kérala indien), des guerriers visiblement Tupinambas (côte sud-est du Brésil) sont illustrés sur les gravures, présentant leurs attributs traditionnels : «jupe » et ornements de plumes, massues de bois.

Un spectacle italien, Il Ballo per Carlo $V$, doit être également cité. Créé semble-t-il à Milan en 1521 par Giulio Romano en l'honneur de Charles Quint, ce ballet à entrées aurait représenté plusieurs personnages exotiques, dont un «Indien» (Martini, 2011). Cependant, en l'absence d'informations complémentaires, il est difficile d'intégrer avec certitude dans l'inventaire un spectacle dont l'Indien pourrait être en fait oriental.

La plus ancienne représentation dont on puisse être certain est en fait créée en 1527 à Dieppe dans des circonstances assez particulières. Cette année-là, l'armateur Jean Ango, donna une fête au cours de laquelle un «masque » intitulé Les Biens fut joué, composé par Jean Parmentier l'un de ses capitaines. Selon les sources, les «Richesses de la terre y étaient déployées et parmi elles figuraient une partie des trésors mexicains envoyés par Cortès à Charles Quint et qui avaient été interceptés par le corsaire Jean Fleury en 1522 non loin des Açores ${ }^{5}$.

Ainsi, pour la première fois un spectacle mettait paradoxalement en scène non seulement une Amérique caricaturée et encore mal définie mais des parures et des costumes « authentiques » issus du monde aztèque ${ }^{6}$.

Il faudra attendre plus de vingt-cinq ans pour assister au spectacle suivant mettant en scène des Américains : l'Entrée royale d'Henri II à Rouen ${ }^{7}$. Célébré à l'occasion de la visite du roi dans la capitale normande, le 1er octobre 1550 , il fut l'occasion de réaliser un fastueux cortège par lequel le roi et sa cour étaient reçus dans la cité et auquel devaient être associés un défilé de chars puis une représentation théâtrale (traditionnellement religieuse). L'originalité de cette dernière réside ici dans son thème : les Indiens Tupinambas du Brésil, mis en scène de manière particulière.

Sur une prairie spécialement aménagée au bord de la Seine et face à la ville, avait été composée une sorte de forêt tropicale notamment par l'ajout de ramures aux arbres existants, la disposition de troncs peints en rouge (symbolisant le fameux bois-Brésil dont la ville faisait commerce) et le lâché d'animaux exotiques (singes et perroquets). Un village Tupi avait été reconstitué avec sa palissade et ses « Naturelz sauvages freschement apportez »; le nombre de véritables Indiens (une cinquantaine) étant renforcé par quelque 250 marins normands grimés pour l'occasion.

\footnotetext{
${ }^{5}$ Sachons qu'un des deux navires espagnols saisis par Fleury s'échoua sur des récifs au Sud de l'île de Jersey et sombra en emportant une partie du fameux trésor.

${ }^{6}$ Si les sources s'accordent sur la spectaculaire prise de Fleury (et la fin tragique du corsaire, exécuté en Espagne pour fait de piraterie cette même année 1527), les informations relatives à la fameuse fête sont en revanche indirectes : voir Asseline, 1874 (p. 224-231) ; Cahingt, 1980 (p. 93-106) ; Thomazi, 1956 (p. 44-45). Guénin, 1901 (p. 118).

${ }^{7}$ Voir Denis, 1850 et Perrone Moisés, 2008. 
Le spectacle, montrant tout d'abord la vie quotidienne des Tupinambas (danses, chasse, escalade dans les arbres, coupe du bois de braise et commerce avec les Français), fut ensuite poursuivi par la représentation d'une bataille entre les habitants du village et des Indiens ennemis (alliés des Portugais). Il se termina enfin par une naumachie entre un navire français et son rival portugais (ce dernier étant bien entendu coulé).

Les gravures publiées les mois suivant l'évènement brillent curieusement par leur inexactitude alors que, selon les sources, le spectacle avait été respectueux des réalités. Cette réinterprétation n'est pas exceptionnelle dans l'iconographie des mondes exotiques et ne fera par la suite que s'accentuer. Elle peut également être justifiée par les causes même de cette représentation exceptionnelle : non pas la recherche d'une description «ethnographique » avant l'heure mais surtout la mise en avant de la présence française au Brésil et des rapports amicaux avec les populations amérindiennes.

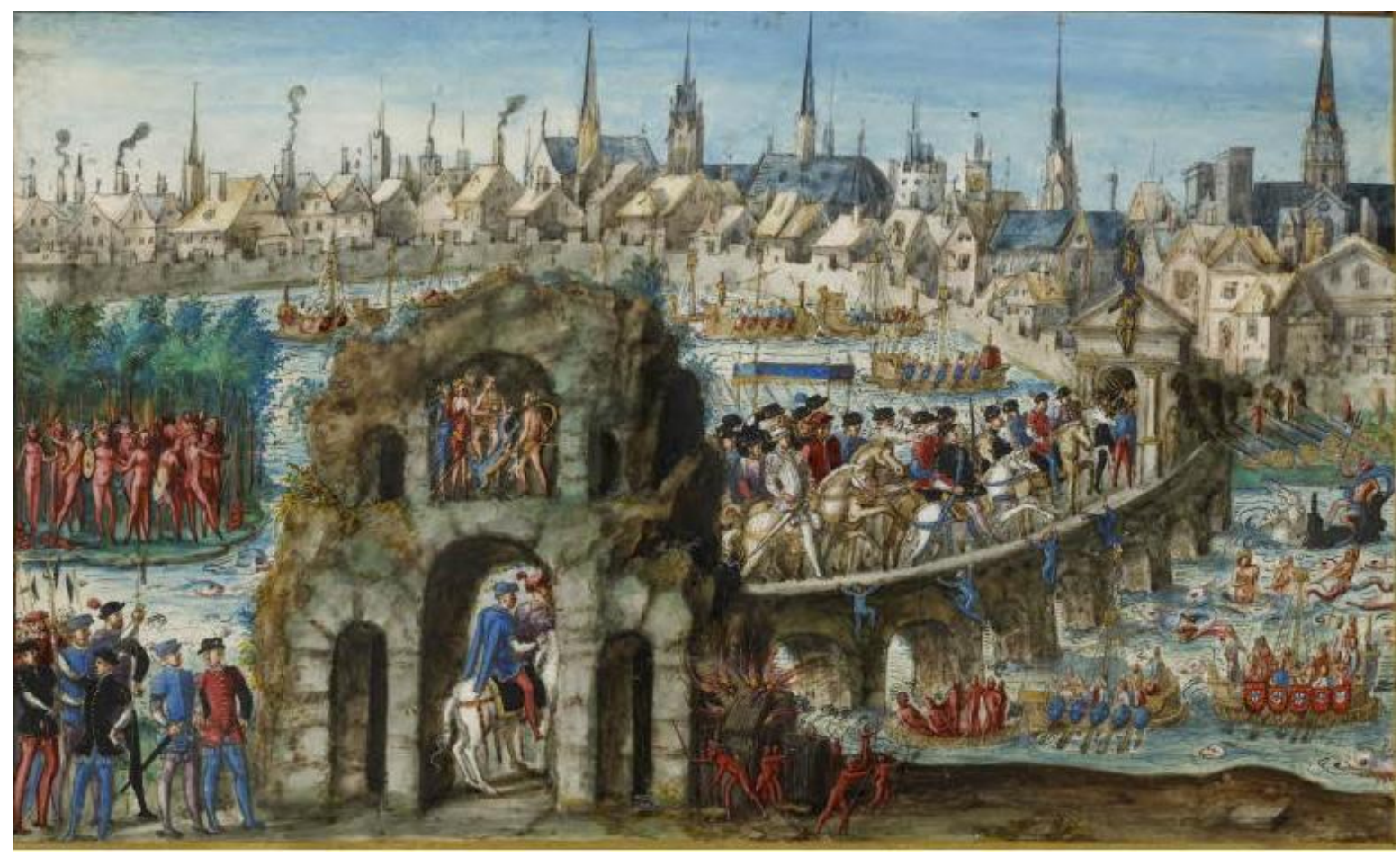

Planche 1. Entrée solennelle d'Henri II à Rouen, le 1er octobre 1550, par Louis Merval.

On distingue les «Tupinambas » dans les bosquets sur la gauche.

Rouen, Bibliothèque municipale

Deux autres entrées royales françaises, plus tardives associant des Indiens d'Amérique peuvent être notées et sur lesquelles peu d'informations ont été recueillies (Denis, 1850, p. 23, note 3 ; Perrone Moisés, 2008, p. 61, note 43) : l'Entrée royale de Charles IX à Troyes, le 23 mars 1564 et l'Entrée royale de Charles IX à Bordeaux, le 9 avril 1565.

Enfin bien que tardifs (à la charnière des XVIe et XVIIe siècles) et chronologiquement liés à la période suivante, trois spectacles germaniques doivent être signalés ici, car appartenant à cette première époque par les sources utilisées et les caractéristiques des spectacles présentés.

Tout d'abord, la Parade nuptiale ${ }^{8}$ de Cassel organisée en 1596 par le Landgrave Moritz dem Gelehrter pour le mariage de sa fille : à ce titre, l'Amérique ouvre le défilé sur des modèles très probablement issus des gravures de Théodore De Bry, fameux graveur et auteur principal de la somme India Occidentalis (ou Grands voyages) éditée à partir de 1590 (Bujok, 2003, p. 82).

\footnotetext{
${ }^{8}$ Faute de titre officiel, nous lui avons attribué celui-ci. 
Ce spectacle a probablement inspiré l'imposant Festaufzug présenté pour le Carnaval, le 25 février 1599, à la cour de Stuttgart sous l'égide du duc Frederick Ier de Württenberg. Königin Amerika fut certainement l'une des parades plus spectaculaire du XVIe siècle puisqu'elle impliqua quelque 6000 participants de toutes conditions en une suite d'entrées dans lesquelles le Nouveau monde joue un rôle majeur, notamment en ouvrant le défilé et auquel le duc en personne prit part (Bujok, 2003). Documentée par un texte de Jacobs Frischlings, publié en 1602 et surtout illustrée par huit aquarelles anonymes ${ }^{9}$, cette entrée est bien connue et nous permet de considérer son organisation et les costumes et parures des participants. L'Amérique, abritée sous un dais est entourée de musiciens et de guerriers et accompagnée par des suivantes portant dans des paniers les richesses naturelles du Nouveau monde. Également inspirée des gravures de Théodore de Bry, cette iconographie présentant pêle-mêle des Brésiliens et des Floridiens tout droit sortis de l'imagination du graveur de Francfort, illustre cependant des parures et des objets particuliers : coiffes et capes de plumes Tupinambas, éventails, chimalli et tlahuiztli aztèques également en plumes; objets bien réels, appartenant vraisemblablement au collections ducales et, sinon vraiment portés, du moins copiées fidèlement ${ }^{10}$.

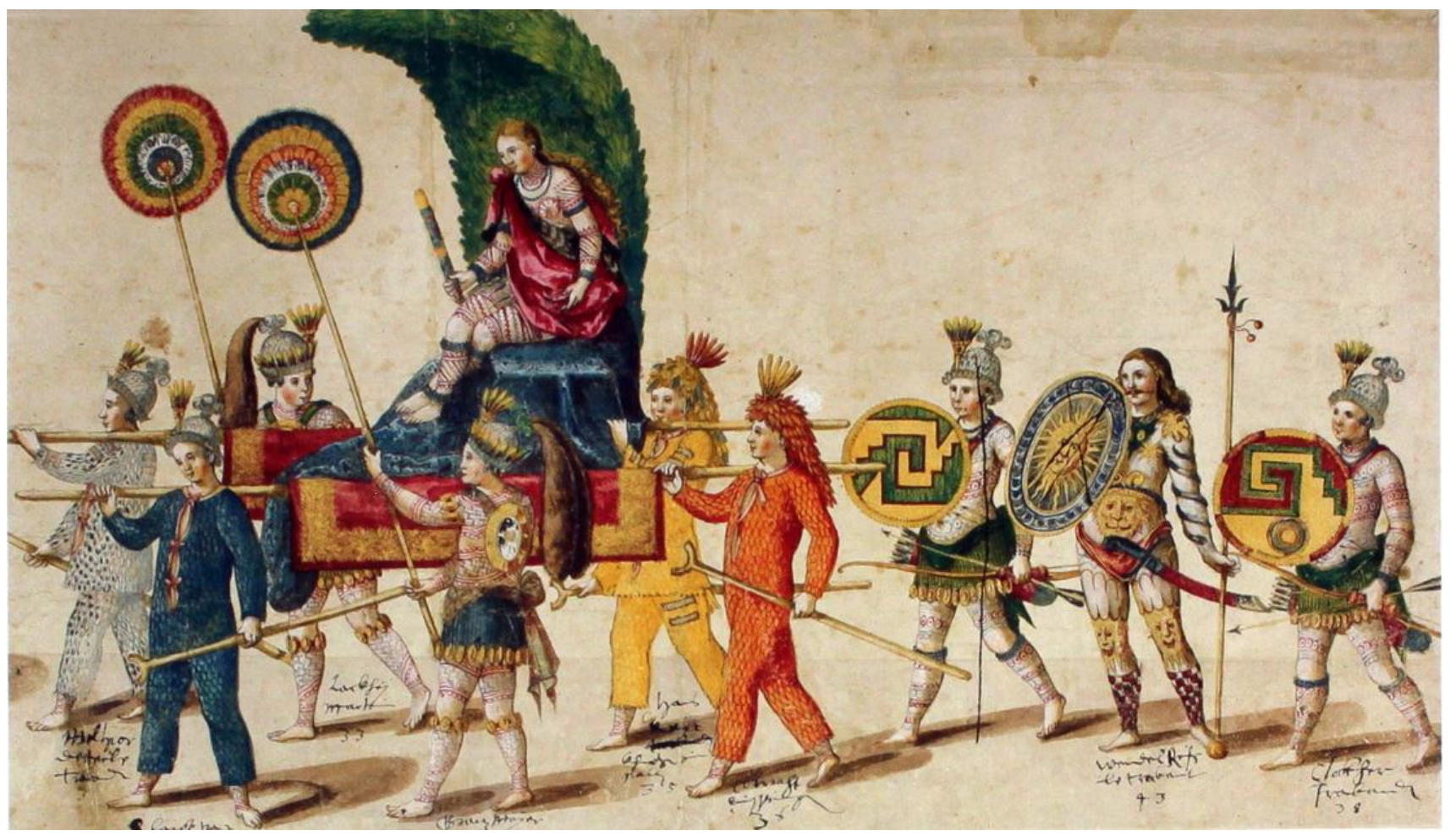

Planche 2. Königin Amerika. Feshauzug organisé pour le carnaval le 25 février 1599 à Stuttgart. Les porteurs de la litière de la Reine des Amériques semblent vêtus de tlahuiztli et les guerriers de l'escorte arborent des chimalli. Dessin anonyme, 1598-99, Weimar, Klassik Stiftung, Graphische Sammlung.

Ce grand spectacle inspirera certainement la Parade nuptiale ${ }^{11}$ organisée dans la même cité quelques années plus tard, en 1609, pour le mariage de Johan-Friedrich (fils ainé de Frederick) : des « Américains » animèrent à cette occasion la dixième entrée (Bujok, 2003, p. 81).

\footnotetext{
${ }^{9}$ Aujourd'hui au Klassik Stiftung de Weimar.

${ }^{10}$ Le chimalli est le bouclier aztèque, accompagnant toujours le tlahuiztli, espèce de justaucorps. L'un et l'autre sont des éléments de parade et de combat, renforcés et toujours couverts de plumes. En grand nombre, ils furent rapportés en Europe. Si plusieurs chimalli existent encore de nos jours (notamment au WürttembergischesLandesmuseum de Stuttgart), aucun tlahuiztli n'est apparemment parvenu jusqu'à nous.

${ }^{11}$ Faute de titre officiel, nous lui avons attribué celui-ci. 


\section{Deuxième époque (XVIle siècle) :}

\section{Les entrées de Sauvages et d'Amériquains}

Après une absence de plus de trente ans (si l'on excepte les trois spectacles germaniques cités plus haut), l'Amérique réapparait sur les scènes et va connaître tout au long du XVIIe siècle un certain succès et surtout un spectaculaire développement tant géographique que chronologique. Trente-sept spectacles peuvent être ainsi comptés, tant en France qu'en Italie et en Angleterre (y compris en Allemagne et au Portugal). Cette présence connaît même son apogée entre 1657 et 1669 avec plus d'une douzaine de représentations.

Cependant, cette évolution peut sembler surprenante en cette époque de relatif oubli du Nouveau monde par la Vieille Europe (isolement de l'Amérique ibérique sévèrement gardée par l'Espagne, lente et difficile colonisation du nord du continent par l'Angleterre et la France, rivalités coloniales exacerbées). À moins qu'elle n'en soit le résultat, par ses caractères : ignorance des cultures qui avaient surpris les élites du siècle précédent, non référence aux objets ethnographiques des cabinets de Curiosités qui avaient jadis été tant utilisés (peut-être disparus ou détruits), création d'une vision iconographique caricaturée fondée sur des lieux communs aisés à concevoir (exotisme, plumes, sauvagerie).

Cette Amérique chantée et dansée, comme pour les autres formes d'arts, prend l'aspect de son temps, celui du Baroque et tient désormais une place particulière dans les entrées et défilés de ballets royaux et les quadrilles de parades équestres : allégories de peuples ou de terres associées à la mythologie classique et aux grandes figures du temps, au sein de décors et de situations sans grand rapports avec la réalité anthropologique.

Cette vision réinventée est la forme choisie par les productions françaises - les plus nombreuses (19 spectacles) - s'échelonnant entre le Ballet à la reine [Paris, 1609] et Issé [Destouche/Houdar, Fontainebleau, 1697] ${ }^{12}$. Les thèmes traités sont variés et évoluent avec le temps, au gré des modes et de l'actualité ${ }^{13}$ :

- Histoires galantes comme dans la Mascarade de Sauvages [Vital, 1614] où l'on découvre des Américains amateurs du Beau sexe déclarant leur flamme aux dames de la Cour.

- Plantes particulières, avec le Ballet de Monseigneur le Prince dansé à Bourges [1621] dans lequel un « fol Indien » se plaint des effets du pétun (le tabac).

- Ravages de l'âge, abordé dans le Grand Bal de la Douairière de Billebahaut [Paris, 1626] où les Nations (dont les Américains) rendent hommage à sa beauté fanée, par les spectaculaires costumes de Daniel Rabel.

Ces sujets légers semblent avoir laissé la place à des thèmes plus sérieux à partir du second tiers du siècle, qu'ils fussent politiques, diplomatiques ou militaires :

- Ballet du Bureau de rencontre dansé au Louvre devant Sa Majesté [1631], œuvre visiblement inspirée par Richelieu, présentant l'état du monde par une chorégraphie de « masques » au cours de laquelle apparaît un Indien canadien ; probablement la plus ancienne représentation en spectacle d'un habitant de cette région du continent.

- Ballet de la Félicité [1639] donné pour la naissance du futur Louis XIV, où les 4eme et 5eme entrées magnifient les victoires de la France sur l'Espagne et où les Nations présentent leurs hommage au roi de France (parmi lesquelles des Péruviens et des Brésiliens).

\footnotetext{
12 [Compositeur/Librettiste, lieu de création, date de création]

${ }^{13}$ Voir Moureau, 2004, 2005.

(c) 2021 ISTE OpenScience - Published by ISTE Ltd. London, UK - openscience.fr
} 
- Temple de la Paix, [Lully/Quinault, Fontainebleau, 1685] dont l'avant-dernière entrée, « des Sauvages de l'Amérique », fait références aux possessions françaises sur le continent.

Enfin, dès le milieu du siècle, peut-on assister à la résurgence des personnages historiques américains et à leur culture, absents depuis longtemps, mâtinée bien entendu des approximations imposées par l'époque et ses goûts :

- Divers entretiens de la Fontaine de Vaucluse [Avignon, 1649], ballet présentant entre-autres un «Grand et inouï Pachacamac ${ }^{14}$

- L'Amour malade [Lully/Bensérade, Paris, 1657], ballet dont la 8eme entrée voit l'apparition d'Atabalipa, roi du Pérou (en fait Atahualpa).

Cependant, de tous ces spectacles, le plus impressionnant fut sans conteste le fameux Grand carrousel royal, donné les 5 et 6 juin 1662 aux Tuileries pour la naissance du Dauphin ${ }^{15}$. Réunissant quelque 1300 participants (dont la fine fleur de la noblesse) devant plus de 10000 spectateurs, il fut composé de joutes équestres et des parades de cinq quadrilles représentant les peuples du monde. La dernière, «Les Sauvages amériquains », conduite par le Duc de Guise rassemblait 160 participants donc 90 cavaliers. Les gravures par Sylvestre et Chauveau, ainsi qu'une description laissée par Perrault nous permettent de mesurer la richesse de costumes baroques bien peu fidèles aux traditions vestimentaires des Amérindiens ${ }^{16}$.

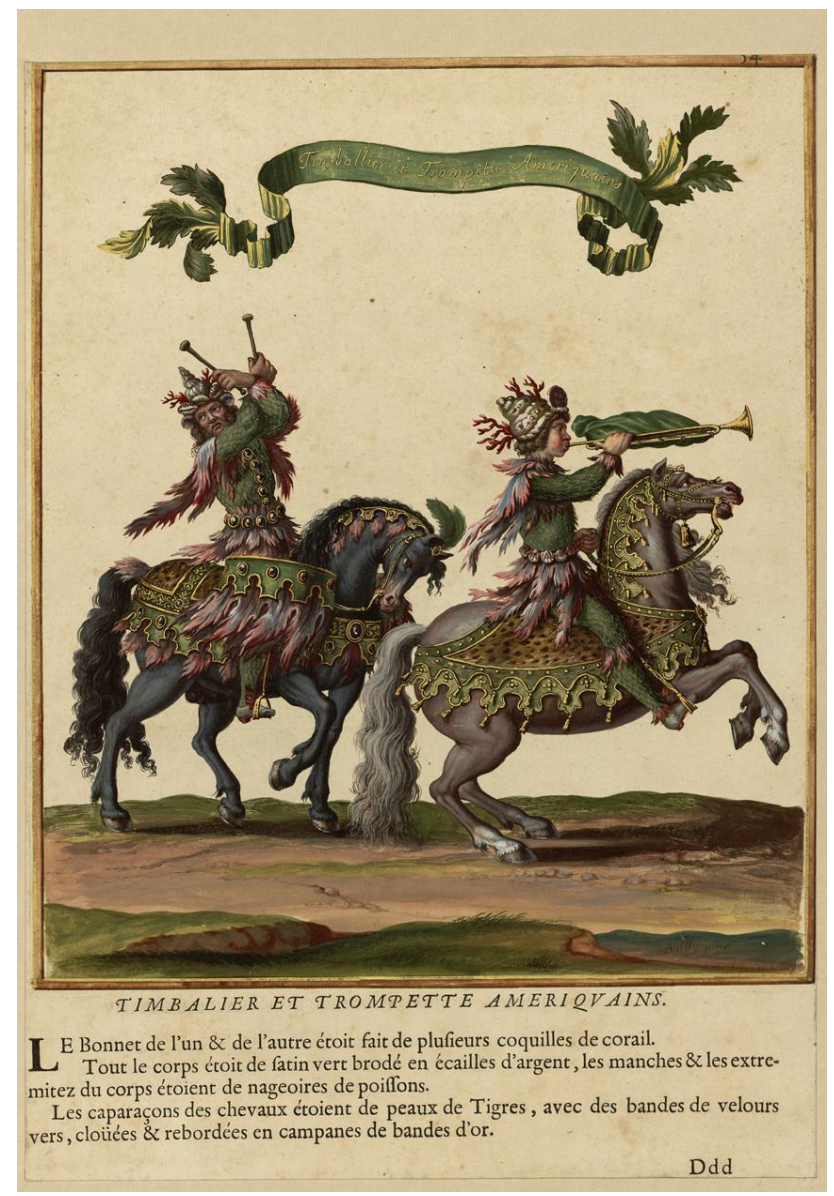

Planche 3. Grand Carrousel des 5 et 6 juin 1662 : "Timbalier et trompette amériquains ». Costumes de François Chauveau, gravures d'Israël Silvestre (1670). Les tenues des personnages semblent fortement inspirées des tlahuiztli aztèques, et dont peut-être quelques exemples existaient encore au milieu du XVIIe siècle. Versailles, Bibliothèque municipale.

\footnotetext{
${ }^{14}$ Pachacamac est en fait un des plus importants sanctuaires précolombiens du Pérou.

${ }^{15}$ Voir Lacroix, 1976.

${ }^{16}$ Sachons que les membres de la quadrille précédente, "Les Indiens » (de l'Inde), arboraient en revanche plumes et perroquets. 
Très proches par leur nature de leur soeurs françaises, les créations italiennes sont cependant moitié moins nombreuses. Cette disparité peut être expliquée par les sujets des spectacles italiens plus volontiers tournés vers l'Antiquité classique.

Des 10 spectacles comptés, citons principalement Il Tabacco [1650], ballet créé à Turin par Filippo d'Agliè (Grammeniati, 2010, p. 91-94). Entièrement consacrée au tabac dont l'usage se répandait alors en Europe occidentale, l'oeuvre est aujourd'hui connue par les spectaculaires illustrations laissées par Tommaso Borgonio représentant les Naturels, ornés de plumes et tenant en mains pipes et mèches. L'unité thématique, très rare pour l'époque, annonce les œuvres du siècle suivant (McGowan, 1972).

Certainement plus spectaculaire encore fut L'unione perla peregrina Margherita reale e celeste, créé également par d'Aglié et dont les costumes sont aussi attribués à Borgognio. Présenté en l'honneur des noces de Marguerite de Savoie et de Ranuccio Farnese, ce ballet se déroula durant cinq heures le 11 mai 1660 dans l'enceinte du Palais San Giovanni de Turin. L'Amérique y figura en bonne place, reconnaissable, bien que son identité de soit pas explicitée (Honour, 1975, p. 103).

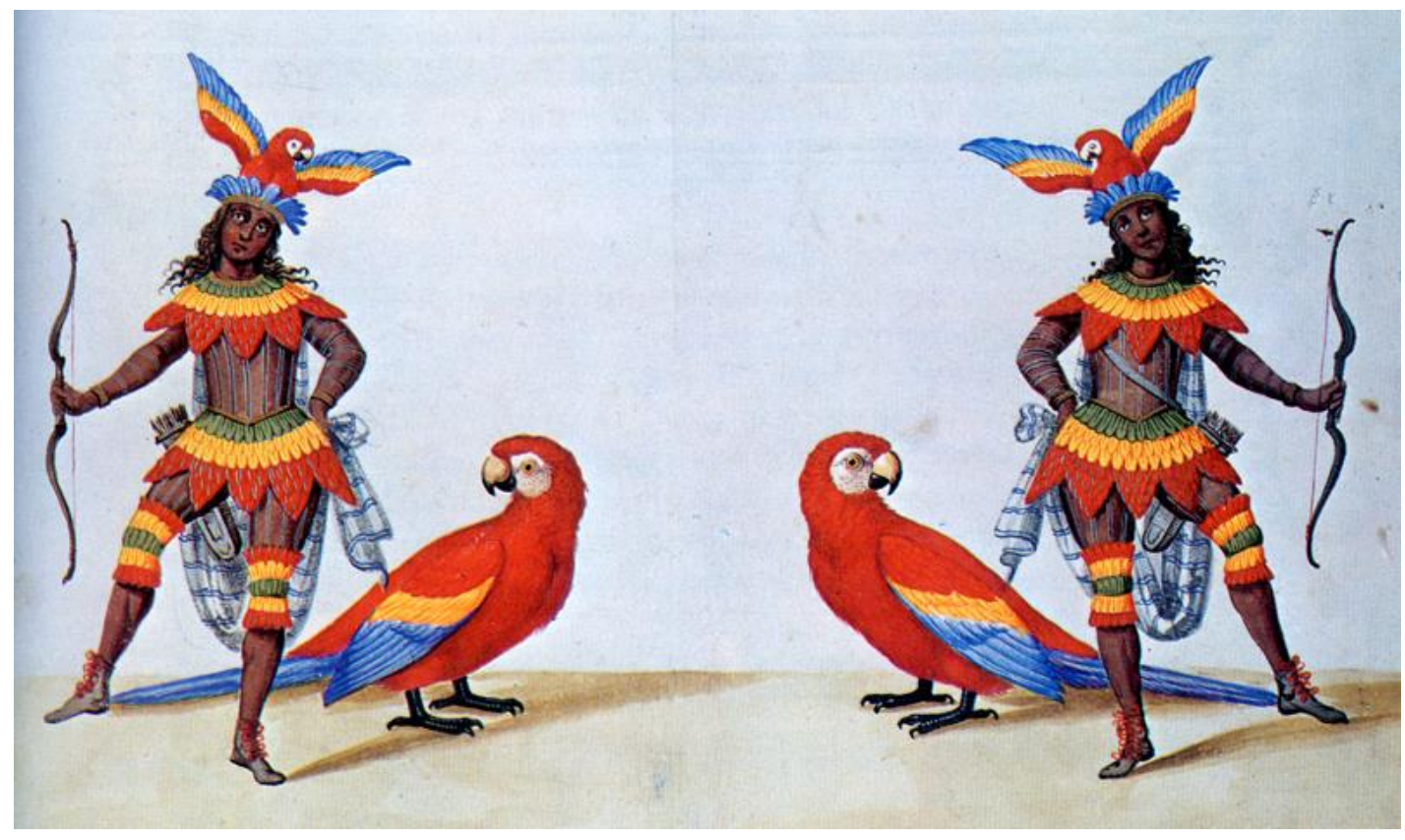

Planche 4. Grand ballet de l'Unione perla peregrina Margherita reale e celeste, Livret et chorégraphie de Filippo d'Aglié, costumes de Tommaso Borgognio. Turin, 11 mai 1660. Entrée des Maestri de i papagalli.

Bien qu'attribués à «Calicut » et portant des noms orientaux, les personnages font ici clairement référence aux Tupinambas par leurs atours et les perroquets (Aras ?) qui les accompagnent.

Turin, Biblioteca nazionale universitaria.

Egalement précurseurs, quatre spectacles doivent être notés: Il Giudizio di Paride («Le Jugement de Pâris ») de Buonarroti, donné à Florence pour le mariage de Cosimo de Médicis en 1608 (Boorsch, 1976, p. 510 ; Tkaczyk, 2016, p. 103-107) ; Le Mettamorfosi d'Amore de Mascardi, créé à Gênes en 1623 (Apollonio, 2014) ; Il Colombo overo l'India scoperta [Pasquini/Ottoboni], donné à Romme en 1690 (Viale Ferrero, 1992, p. 60) ; enfin un peu plus tardif mais de la même veine Il Colombo, présenté à Gênes en 1708 (Heck, 1992, p. 238 et 252). Si les deux premiers permettent respectivement l'apparition de Vespucci et de Colomb, les deux derniers (comme leur nom l'indique) sont entièrement consacrés au navigateur génois et annoncent les représentations hagiographiques (ou critiques) que l'on verra beaucoup plus tard (XIXe et XXe siècles).

Bien différentes des spectacles latins, les représentations anglaises du Nouveau monde durant le XVIIe siècle sont marquées par une approche beaucoup plus politique (Frohock, 2001, passim). Des six œuvres connues - et si l'on excepte The Middle Temple Mask [Chapman, 1613] proche des 
entrées françaises et italiennes et peut-être la célèbre Indian Queen [Purcell /Dreyden et Howard, 1695] - toutes sacrifient à la Légende Noire qui se développait depuis l'extrême fin du XVIe siècle et à laquelle les élites anglaises adhéraient avec passion. Le titre de la première est révélateur : Cruelty of the Spaniards in Peru, en 1658 [Locke/Davenant]. Son livret inspira d'ailleurs les autres productions : History of Sir Francis Drake, en 1659 [Locke/Davenant], Indian Queen en 1664 [Humfrey/Dreyden et Howard] et Indian Emperor en 1665 [des mêmes]. Cette vision antiespagnole, voire anti-catholique, fera florès bien plus tard en Europe à la fin du XVIIIe siècle.

Signalons enfin, pour clore cette période, deux œuvres particulières, tant par leur origine que par leur thème : la Fête en l'honneur de la canonisation d'Ignace de Loyola, donnée à Lisbonne en 1623, dont l'un des chars représentait l'Amérique (Boorsch, 1976, p. 507) ; et surtout Il Pomo d'Oro [Cesti/Sbarra], joué à Vienne en $1668^{17}$. Cette création italienne fastueuse, commandée par l'Empereur Léopold pour son mariage avec l'infante Marguerite d'Espagne (et que nous associons pour cette raison au monde germanique) est fondée sur le mythe de la Pomme de Pâris. L'Amérique y apparaît au sein du prologue. ${ }^{18}$

\section{Troisième époque :}

\section{L'apogée "américaniste " dans les spectacles chantés et dansés des XVIIle et XIXe siècles}

Si l'on compte les quelques œuvres de la fin du siècle précédent, signalées plus haut, et celles également peu nombreuses - de la première moitié du XVIIIe siècle, nous pouvons constater un fort recul des spectacles chantés et dansés abordant les thèmes américains entre 1670 et 1760 (soit presque cent ans), alors que les spectacles musicaux et chorégraphiques ne cessaient de croître en nombre.

Entre 1720 et 1760 seuls huit opus «américanistes » peuvent être notés. C'est pourtant durant cette période que furent créés les deux spectacles certainement les plus connus du répertoire «américaniste»: Motezuma [Vivaldi/Giusti] donné à Venise en 1733 et Les Indes galantes [Rameau/Fuzelier] présenté à Paris en $1735^{19}$. Cependant, ces deux œuvres, aujourd'hui célébrissimes, n'auront en fait que peu d'impact sur la production du genre : Motezuma, oublié rapidement n'est certainement pas à la source de l'intérêt que le personnage développera durant la seconde moitié du siècle ; quant au Indes galantes, malgré le succès qu'elles connaîtront jusqu'en 1761 (185 représentations), elles disparaîtront presque complètement de la scène jusqu'en $1952^{20}$.

C'est donc dès 1760 que commence une longue période qui verra l'apogée du spectacle musical et dansé «américaniste» et qui durera jusqu'à la veille de la Première Guerre mondiale: impressionnante production rassemblant plus de 280 œuvres au rythme d'une quinzaine de spectacles par an (au minimum) pour toute l'Europe. Les productions italiennes et françaises y furent les plus nombreuses avec respectivement quelque 110 et 70 créations $^{21}$. La majorité des œuvres « américanistes » de ces deux pays apparaît durant cette époque.

Cette dernière apporte en fait une grande nouveauté : pour la plupart ces créations sont entièrement consacrées au Nouveau monde. Les titres d'ailleurs en font foi, définissant clairement des lieux, des personnages et des situations qui ne laissent aucun doute. Les causes, outre l'orientation «politique » plus grande des livrets, résident également dans l'évolution technique et

\footnotetext{
${ }^{17}$ Voir Schmidt, 1976.

18 Information tirée de l'inventaire de Pisani.

${ }^{19}$ Les 2e et 4e « entrées » sont consacrées aux Amériques : Les Incas et Les Sauvages. Voir Savage, 1983.

${ }^{20}$ Ne sont ici comptées que les représentations complètes.

${ }^{21}$ Signalons également 47 créations germaniques, 31 anglaises, 16 espagnoles, 2 belges, 2 russes, une suédoise, une danoise, une polonaise, une hongroise, une portugaise et une tchèque. 
artistique des spectacles : non plus ballets-royaux à entrées indépendantes mais opéras et ballets à thèmes, inaugurant ce qui allait être désormais la règle. ${ }^{22}$

Ces thèmes, en somme peu nombreux, connaitront un grand succès et se répandront jusqu'à nos jours. Outre les champs traditionnels déjà connus depuis longtemps (exotisme, honneur, amour, gloire du prince), apparaissent de nouveaux sujets de livrets jusqu'alors peu ou jamais abordés : les Découvreurs (principalement Colomb), l'exploration, les conquêtes du Mexique et du Pérou, la Nouvelle-France, l'Amérique anglo-saxonne (période coloniale, guerre d'Indépendance et conquête de l'Ouest), l'esclavage.

Cependant, cette riche époque n'est pas homogène, durant laquelle en effet les thèmes et les orientations évoluent. En somme, deux périodes doivent être considérées, dont la charnière est la chute de l'empire et la fin des guerres napoléoniennes.

\section{Des années 1760 à la fin de l'Empire}

Cette période, relativement courte (une cinquantaine d'années), apparaît comme extrêmement fertile pour la production de spectacles « américanistes », très certainement à l'instar de l'ensemble des créations lyriques et chorégraphiques alors en pleine mutation, reflets fidèles des bouleversements dans lesquels elles apparaissent.

Bien curieusement d'ailleurs, malgré les troubles et les guerres engendrées par la Révolution et l'Empire - peut-être même à cause de ceux-là - leur nombre connaît entre 1780 et 1800 un accroissement spectaculaire, véritable apogée avec 80 créations (dont 34 italiennes, 19 françaises, 14 germaniques et 9 anglaises).

En fait, l'origine du nombre surprenant de productions «américanistes » apparaissant dès 1760 est certainement à rechercher dans Alzire [1733], tragédie d'un Voltaire encore jeune ${ }^{23}$. Les sujets abordés : le dramatique destin de l'empereur des Incas et la Conquête espagnole vont, durant les décennies à venir, orienter la vision européenne des Amériques vers une image plus grave, plus sombre, assurément politisée et dénonciatrice, dans la droite ligne du combat des Lumières.

Curieusement, les premiers sujets abordés dans les livrets ne sont pas péruviens mais mexicains : confusion favorisée par les connaissances alors bien minces sur le monde précolombien. Les noms des personnages principaux en sont la preuve, indifféremment aztèques ou incas, selon les besoins des intrigues.

Montezuma de Graun [Berlin, 1755] est assurément le précurseur de cette vague « mexicaniste » et dont le livret écrit en français par Frederick II de Prusse est directement inspiré de la tragédie de Voltaire. L'œuvre, volontiers anti-espagnole, marquera durablement les esprits quelques années plus tard (Nuñez Ronchi, 2014, p. 498-500) ${ }^{24}$. Entre 1765 et 1810 , près de 25 créations sur le thème apparaissent, inspirées les unes des autres et dont seuls les titres changent : «Montezuma » ou «Motezuma» (voire «Montesuma»), «La Conquête du Mexique », «Fernand Cortez» ou «Hernán Cortès », « Sicotencal » ${ }^{25}$, «Irza », «Les Mexicains », « Amazilia » etc. Les œuvres italiennes sont de loin les plus nombreuses (14). Quatre sont espagnoles, trois françaises, deux

\footnotetext{
${ }^{22}$ Les entrées américaines, souvent dansées, incluses dans des œuvres sans rapport avec le Nouveau monde existeront cependant jusqu'au début du XIXe siècle, comme pour l'opéra Gli Arabi nelle Gallie (1828) abritant un ballet intitulé La Conquista del Perú.

${ }^{23}$ Voir Sanchez, 1992.

${ }^{24}$ Voir également Duviols, 1997.

${ }^{25}$ En fait Xicotencatl, général et grand guerrier farouchement opposé aux Espagnols alors que sa cité (Tlaxcala) était alliée aux conquistadors dans leur lutte contre Tenochtitlan, la capitale des Aztèques. Exécuté par Cortès, il est devenu au XIXe siècle un symbole du nationalisme mexicain. 
anglaises et une germanique. Si cette distribution latine prédominante peut expliquer le rôle majeur que jouent l'amour et la trahison, comme pour Sicotencal ou Irza, elle justifie plus encore la vision plutôt bienveillante que l'on peut noter dans les livrets, bien que les excès de la Conquête et la grandeur d'âme des vaincus y soient également présents.

Certaines œuvres font même apparaître Cortès comme un héros digne des romans de chevalerie. C'est le cas des opéras espagnols, pour des raisons aisées à comprendre ${ }^{26}$. C'est aussi le cas du Fernand Cortez de Spontini (Paris, 1809), créée dans des conditions particulières et dont on dit que le livret d'Esmenard et de Jouy fut peut-être «inspiré » par Napoléon entamant alors l'invasion de l'Espagne. Il s'agissait pour l'Empereur, à travers une intrigue touffue accentuant la noblesse d'âme et le courage des conquistadors (et inversement stigmatisant la férocité des Aztèques), de montrer par association d'idée - le bien fondé des campagnes militaires françaises dans la péninsule ibérique. La réaction du public, interprétant le livret au pied de la lettre, conduisit les autorités à faire interrompre les représentations qui ne reprendront qu'après la chute de l'Empire.

Le thème s'essoufflera par la suite puisqu'on ne trouvera qu'une douzaine de titres. entre 1819 et la veille de la première Guerre mondiale.

Alors que les thèmes «mexicanistes » se répandaient sur les scènes, les œuvres consacrées au Pérou en étaient curieusement absentes ${ }^{27}$. Leur apparition, plus tardive, est en fait liée à la publication des Incas ou la destruction de l'Empire du Pérou de Marmontel (1777). Sa tragédie connaissant un immense succès (bien plus encore que celle de Voltaire), fut a la source d'un engouement immédiat et d'une vague de production de spectacles lyriques sans précédent. Dès l'année de sa parution, est créé à Florence Alzira [Rutini/Oliveri] - notons le titre voltairien - et surtout l'année suivante, à Munich, Kora und Alonso [Winter/von Babo].

Dés lors, une suite ininterrompue de créations, inspirées fidèlement de l'oeuvre de Marmontel se succéderont, aux livrets fort semblables. Les titres en portent la marque car, outre «Alzira» ou «Alzire», nous trouvons : «Cora et Alonso », «Kora und Alonso », «Idalide ${ }^{28}$, «Pizzaro nell'Indie », «Gonzalvo », «La Vergine del Sole », «The Virgin of the Sun », «Les Vierges du Soleil », « La Morte di Pizarro », « Les Péruviens », etc.

Entre 1777 et 1812, plus de 55 spectacles sont ainsi donnés (notamment 6 en 1797), rassemblant la plus forte concentration sur un même thème de tout l'inventaire. De cet ensemble, les créations italiennes sont les plus nombreuses (26), dominant de loin des créations germaniques (12) et françaises (10). Notons enfin Kora och Alonso [Naumann/ Adlerbeth], donné à Stockholm en 1782 et première production «américaniste » née hors du pré-carré de l'Europe occidentale (Saby, 2008, p. 428-431).

Très curieusement, cette vague «péruaniste» va s'affaisser aussi brutalement qu'elle était apparue, à partir de 1812. Entre cette date et le début de la première guerre mondiale, seuls une dizaine de spectacles consacrés à la conquête du Pérou seront créés. Le rapprochement entre ce net recul et la fin de la présence militaire française en Europe est tentant : doit-on voir dans le grand nombre d'oeuvres consacrées à l'occupation du Pérou un symbole de l'occupation française des pays d'Europe ?

Outre les Conquêtes, un troisième thème semble étroitement lié à cette période : celui de l'esclavage. Il ne connaîtra cependant dans les livrets qu'un succès relatif : un peu plus d'une dizaine

\footnotetext{
${ }^{26}$ Des cinq productions espagnoles connues de cette époque, quatre sont effectivement consacrées à la Conquête du Mexique (Arenas Fruto, 1990, p. 284-287).

${ }^{27}$ Signalons cependant le ballet Alzire de Hilverding créé à Vienne en 1742 (Voir Girardi, 1992).

${ }^{28}$ Cora (Vierge du Soleil), sa confidente Idalide, et Alonso l'Espagnol sont les personnages principaux de l'œuvre. 
d'oeuvres y sont consacrées ${ }^{29}$. De celles-ci, la grande majorité est consacrée à la triste histoire de Yariko.

En 1657, A True and Exact History of the Island of Barbadoe, avait relaté une dramatique histoire (vraie selon son auteur, R. Ligon): Thomas Inkle, jeune commerçant anglais échoué sur une île des Caraïbes est sauvé par Yariko, une jeune Indienne. L'un et l'autre tombent amoureux mais lorsque le couple réussit à revenir au port de la Barbade, Inkle vend Yariko qui pourtant attendait un enfant de lui. Le récit avait ému le public tant en Angleterre que dans les pays germaniques et plusieurs romans en avaient été tirés (Kunz, 2007, passim).

C'est donc à partir de ces derniers qu'en 1777 est créé à Dessau Inkle und Yarico [Rust/Schink]. De ce «duodrama», seront issus les spectacles suivants, visiblement fidèles copies présentées jusqu'en 1807 (seuls les titres subissant de légères modifications) ${ }^{30}$. Cette source unique peut sembler bien maigre si l'on admet que les essais philosophiques et littéraires abordant la question de l'esclavage n'étaient pourtant pas rares à cette époque ; choix cependant compréhensible si l'on note que cette question n'est abordée qu'à travers le thème de la trahison de l'amour et non sur le plan de l'Abolition et encore moins de l'égalité des droits. Sachons également - autre particularité de ce thème - que presque toutes ces créations sont germaniques, marque d'un certain intérêt pour le sujet que ne semblent pas partager les autres pays. Signalons néanmoins en 1786 une version française, L'Hérö̈ne américaine [Arnould, Paris], à la fin de laquelle Inkle, capturé par les Indiens après son forfait, est sauvé du supplice par Yariko qui le renvoie avec mépris chez les siens. L'année suivante, un Inkle and Yariko anglais [Arnold/Colman, Londres] proposait un dénouement plus heureux : Inkle se repend et vient rechercher Yariko.

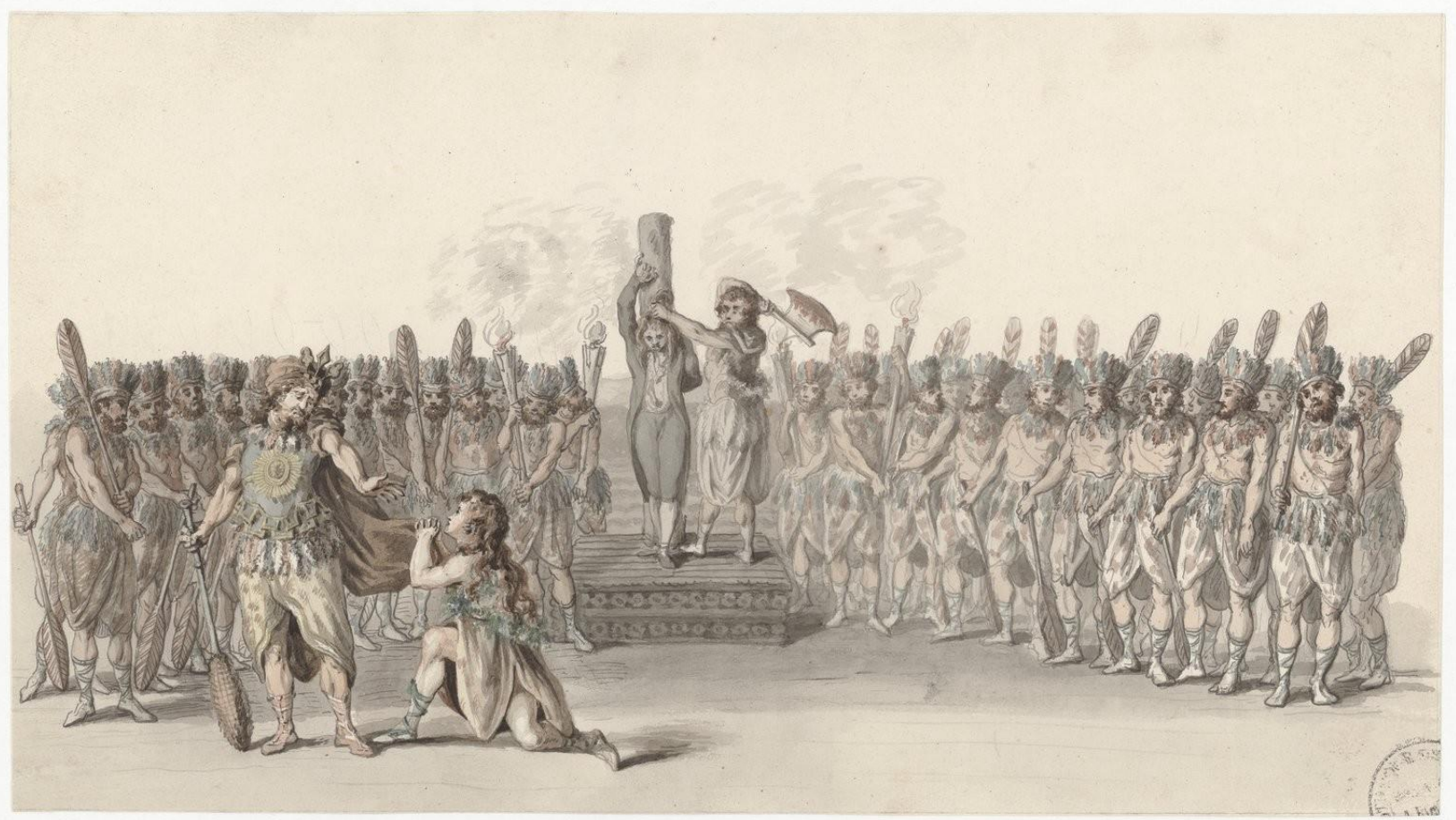

Planche 5. L'héroïne américaine, pantomime de Jean-François Arnould créée le 16 mars 1786 à l'AmbiguComique, Paris. Dessin de l'atelier Ziesenis. Acte III, scène 14 : Yariko intervient pour sauver la vie d'Inkle alors qu'il allait être exécuté. Notons les tenues et les massues «brésiliennes » des guerriers. Gallica BNF.

\footnotetext{
${ }^{29}$ Si les personnages d'esclaves Noirs ne sont pas rares dans les livrets « américanistes », ils ne sont en fait que des éléments secondaires aux intrigues consacrées à bien d'autres thèmes.

${ }^{30}$ Huit opéras relatant la triste histoire de Yarico peuvent être comptés, auxquels doit être ajoutée une ultime version créée à Londres en 2015. 
Enfin, une autre création française particulière doit être notée, Adonis ou le bon Nègre [Beraud et Rosny, Paris, 1798], dont le livret, issu du roman éponyme de Picquenard (publié la même année), a pour toile de fond la grande révolte des esclaves qui ensanglanta l'île de Saint-Domingue (alors territoire français) : Adonis, le «bon Nègre » sauve son maitre et sa famille tombés entre les mains des insurgés. Il les fera évader et tous se réfugieront en Virginie où, selon l'épilogue, ils vivront dans l'égalité et la paix. Outre l'intrigue et sa fin heureuse (de surcroît aux Etats-Unis), est ici planté pour la première fois la question des institutions serviles (Van Bellen, 1927, p. 172).

Elles ne seront pourtant abordées que fort rarement dans les opéras plus tardifs, alors que la question deviendra un véritable sujet d'actualité. Seulement six œuvres peuvent être comptées jusqu'à nos jours, de titres - il est vrai - plus variés et plus «investis »: Le Planteur [Monpou/ Saint-Georges, Paris, 1839] ${ }^{31}$, Le Code Noir [Clapisson/Scribe, Paris, 1842], Toussaint Louverture [Hermann/Lemminger, Lübeck, 1855], Koanga [Delius/Keary, Elberfeld, 1904], Toussaint [Blake/Ward, Londres, 1977], Yariko [McConnell/Miller, Londres, 2015].

La naissance des Etats-Unis apparaît dans les opéras dès la fin de la guerre d'Indépendance. Ils sont cependant relativement peu nombreux ${ }^{32}$ : L'Americana in Olanda [Anfossi/Porta, Venise, 1778], Mirza [Gossec/Gardel, Paris, 1779], La fête de Mirza [les mêmes, Paris, 1781] ; The Fair American [Carter/Pilon, Londres, 1782], L'Orfanella americana [Anfossi/Bertati, Venise,1787], L'Orfanella americana [Gestewitz/Friedrich, Dresde 1790], La Fête américaine [Walter/Peicam, Paris, 1794]. Comme on le voit d'après les titres, l'émancipation américaine n'apparaît ici qu'en toile de fond d'intrigues variées : passion amoureuse pour les deux Mirza (Drawicki, 2002) et surtout pour The Fair American, création particulière au sein de laquelle Angélique, jeune américaine, choisit le camp loyaliste pour l'amour d'un officier anglais (Worrall, 2014, p. 45). Signalons également les deux Orfanella, consacrées à la communauté des Quakers qui avait tant impressionné les Philosophes ${ }^{33}$.

\section{De la Restauration à la veille de la Première Guerre mondiale}

La fin des guerres napoléoniennes et le retour de la paix en Europe, ainsi que l'émancipation consécutive de l'Amérique latine doivent être considérés comme une charnière entre deux époques. Le XIXe siècle européen, période de relative stabilité politique et de paix, de développement colonial et industriel, va profondément marquer sa relation au Nouveau monde : redécouverte du continent, arpenté par les peintres voyageurs et les premiers explorateurs (à l'instar de l'Orient), reconnaissance des civilisations anciennes et nouvelle vague de collectionnisme, intérêt pour les sociétés indigènes. Les spectacles illustrant le continent n'échappent pas à cette évolution, certainement favorisée par de nouvelles conceptions scéniques (ballet-pantomime, opérette).

L'image des Amériques en sera profondément modifiée : les grands thèmes philosophiques et politiques (Conquêtes, esclavagisme) chers aux Lumières et à la Révolution sont délaissés au profit d'un retour à l'exotisme faisant la part belle aux explorations, à l'ethnographie naissante, et bien sûr à l'ethnocentrisme voire aux considérations raciales. Cet exotisme sera le principal filon exploité par les librettistes durant le siècle.

Il s'était certes déjà développé durant la période précédente, et avec dynamisme puis que l'on peut compter une trentaine d'œuvres réalisées entre 1765 et 1812, principalement françaises, italiennes et anglaises. Les thèmes en avaient été fort variés, depuis ceux puisant leurs sources dans les

\footnotetext{
${ }^{31}$ Voir Ponzetto, 2016.

${ }^{32}$ Voir Polzonetti, 2011.

${ }^{33}$ Le Gare Generose [Paisiello/Palomba, Naples, 1786] et The Americans [Brahams et King/Arnold, Londres, 1811] y seront également consacrés. Signalons enfin La Quaquera spiritosa (« La Quaker amusante ») [Guglielmi/Palomba, Naples, 1783] " commedia » qui, malgré son titre, ne semble pas faire référence à cette communauté religieuse. 
spectacles pré-révolutionnaires à entrées, comme Les sauvages ou les pouvoirs de la Danse [Gardel, Paris, 1786], jusqu'aux intrigues amoureuses compliquées: New Spain, or Love in Mexico [Arnold/Scawen, Londres, 1790], La Selvaggia del Messico [Nicolini/Prunetti, Rome, 1803], Le Héros américain, [Ribié/Braban, Paris, 1805]. Notons également l'apparition à cette époque du premier ouvrage russe: Amerikantsy [Fomine/Krylov, Saint-Petersbourg, 1800], à la fin duquel les protagonistes réconciliés, Péruviens comme Castillans, se rendent en Espagne (Lischke, 2017, p. 75-79). Une seule de ces créations, par son sujet semble se démarquer de l'héritage culturel du XVIIIe siècle et annonce la recherche "vériste » du siècle a venir: The Cherokee [Storace/Cobb, Londres, 1794], certes histoire d'amour et de rivalité, s'inscrivant cependant au sein du monde amérindien.

C'est donc à partir de 1825 que la grande vague exotique de l'opéra et du ballet « américanistes » se développe. Plus de cinquante-cinq créations peuvent être comptées jusqu'à la fin du XIXe siècle. L'apogée de cette vague se situe entre 1845 et 1870 (une trentaine de titres), avec une majorité de créations françaises (16 œuvres) correspondant principalement à la période du Second Empire et au développement des opérettes. A partir des années 1870, le genre s'essoufflera puis disparaitra dès 1898 pour ne reprendre qu'à partir des années 1920.

Cet «américanisme » exotique (mais également vériste, comme en témoignent les efforts des costumiers et des décorateurs de l'époque), présente des aspects variés, dûs peut-être moins à l'ouverture de la vieille Europe aux richesses culturelles du Nouveau monde qu'à l'imagination visiblement sans limite des librettistes. Les auteurs français brilleront particulièrement dans ce domaine :

- Jocko ou le singe du Brésil [Picini/Rochefort et Lurieu, Paris, 1825] qui, apprivoisé, puissant et intelligent, sauve son petit maître d'un naufrage, et meurt noyé. Rassurons-nous, dès la deuxième représentation le public exigea qu'il survive. Ce premier ballet « exotique » eut un immense succès et fut maintes fois copié : Danina oder Joko der brasilische Affe [Lindpainter, Stuttgart, 1826] ; Jocko ossia la scimmia brasiliana [Lindpainter/Bouquet, Naples, 1828].

- Amazampo ou la découverte du Quinquina [Lemoine et Meyer, Paris, 1835]: intrigue amoureuse se déroulant dans les environs de Lima au XVIIe siècle et dont la redoutable complexité est aggravée par le secret du petit arbre médicinal jalousement gardé par les Incas. Sachons que les amours seront comblées et la racine généreusement distribuée à tous.

- Jaguarita l'Indienne [Halevy/Saint-Georges et Leuven, Paris, 1855], qui met en scène une farouche princesse guyanaise dont l'air : «Je suis la panthère, la Reine des bois » peut inquiéter des colonisateurs hollandais détestés. Mais Jaguarita aime Maurice, le jeune officier qui a su la dompter. Ils se marieront donc.

- Jovita ou les Boucaniers [Labarre/Mazilier, Paris, 1853], ballet-pantomime qui met en scène le courage de la jeune indigène délivrant l'officier qu'elle aime retenu en otage par de féroces boucaniers, en fait bandit de grands chemins dans le livret. Le ballet du troisième tableau reçut les éloges de la presse d'alors. 


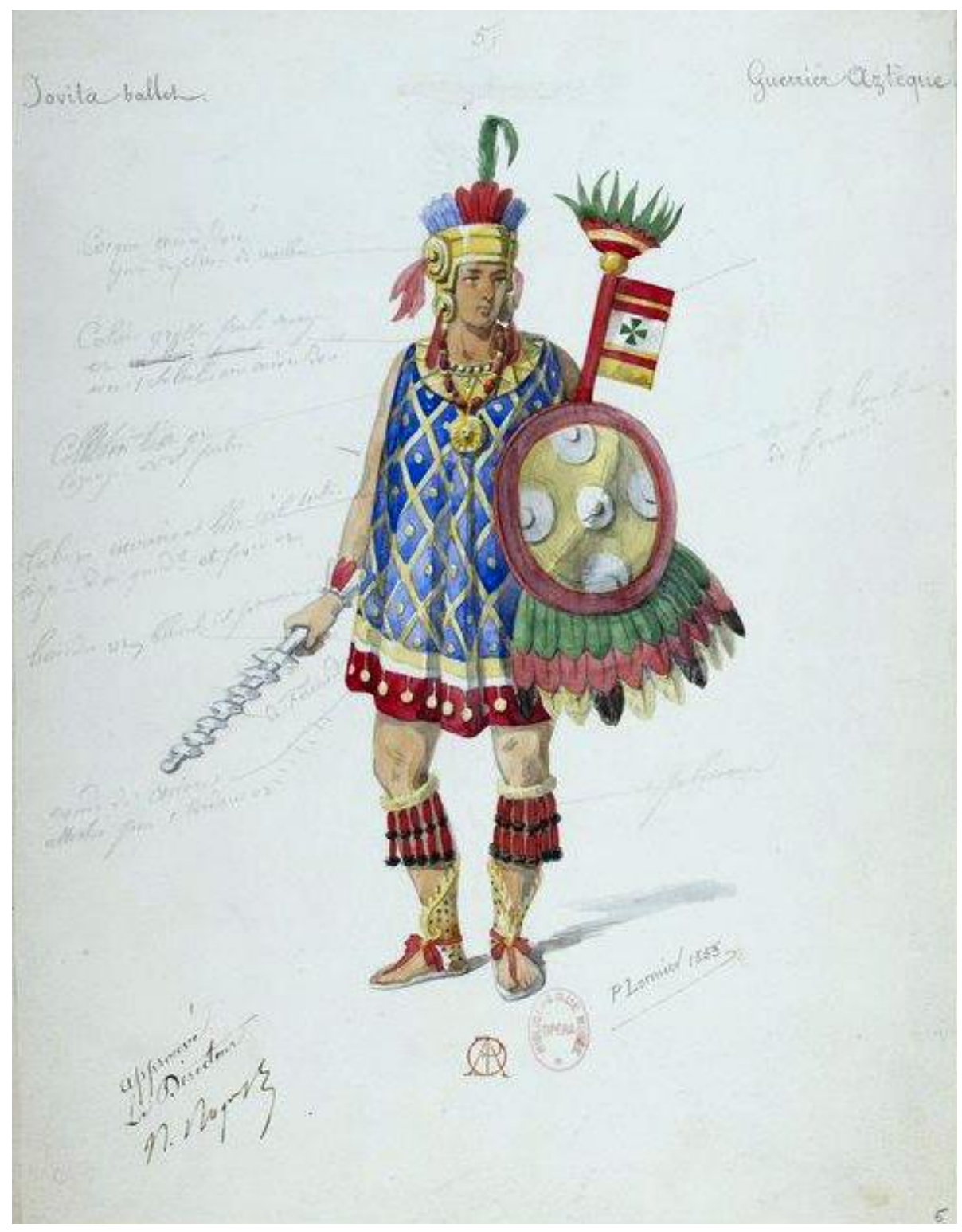

Planche 6. Jovita ou les Boucaniers. Ballet-pantomime donné le 11 novembre 1853 à Paris. " Guerrier aztèque ", appartenant probablement au troisième tableau du ballet : le grand bal donné par le Vice-roi pour fêter la victoire de Jovita sur les boucaniers. Notons la qualité du costume de Lormier qui certainement s'inspira de sources iconographiques que l'on redécouvrait alors. Gallica BNF.

- Le Pays de l'Or [Vasseur/Vanloo et Chivot, Paris, 1892] est en fait la mine californienne que Kitty, riche héritière, essaye de rejoindre en s'évadant de sa pension. Elle affrontera bien des dangers en traversant les Etats-Unis, illustrés par des spectaculaires décors et machineries (chutes du Niagara) répartis sur les 14 tableaux de l'opérette. Apogée dans la démesure et le succès (plus de 140 représentations), elle attira quelques critiques pour sa musique jugée trop facile : «En embarquant pour New-York, on ne songe pas à chanter du Wagner!» (Operette-theatremusical.fr / Vasseur). 


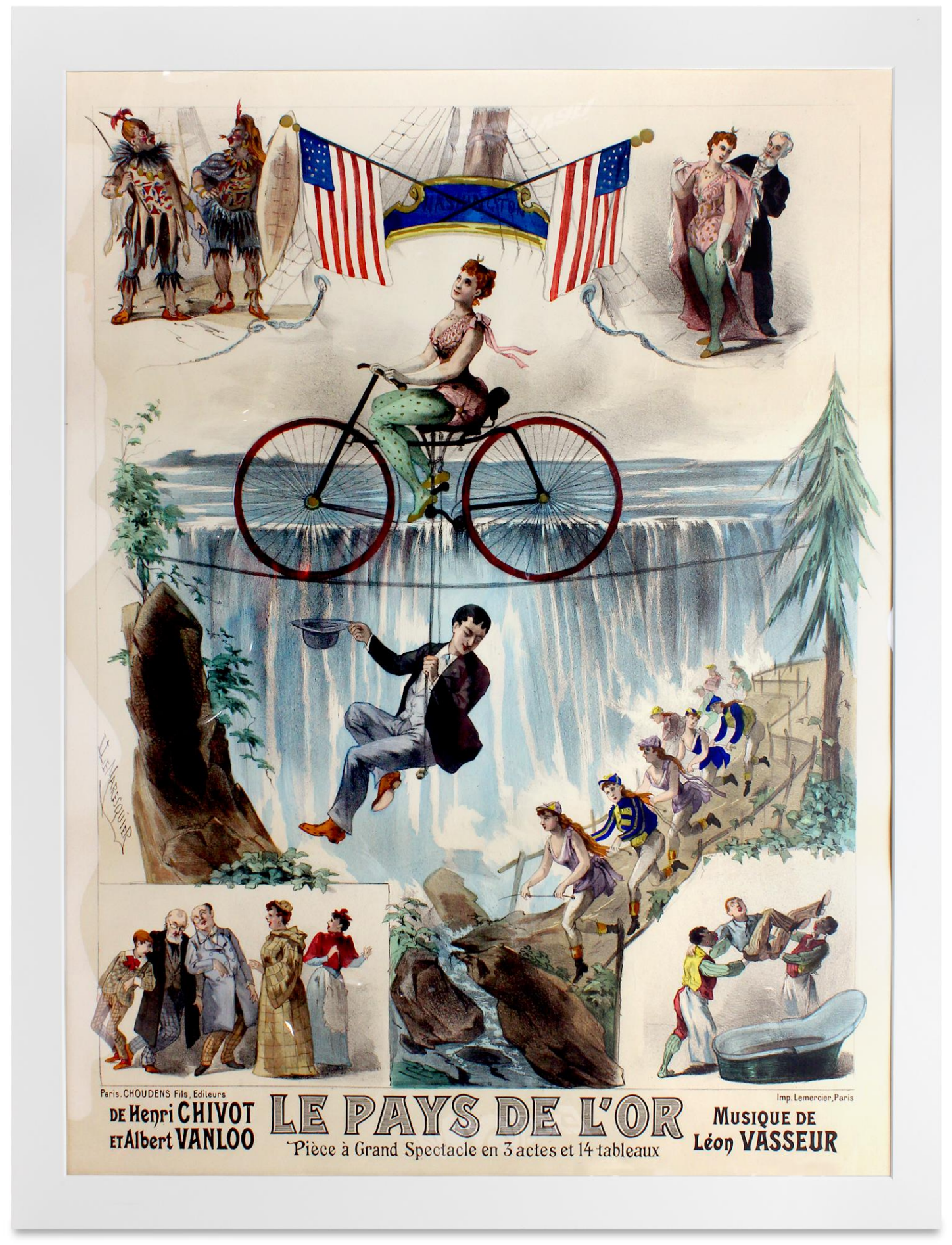

Planche 7. Le Pays de l'Or, «Pièce à grand spectacle avec pantomime... », donnée le 26 janvier 1892, à Paris. L'affiche résume hardiment les multiples épreuves endurées par la courageuse Kitty dans sa quête. Opérette-Théâtre Musical.

Enfin, les plus célèbres de toutes sont sans conteste les créations d'Offenbach qui n'hésita pas à commettre plusieurs américaineries $^{34}$ : Le Brésilien (1863), La Périchole (1868) et La Créole (1875), sans compter la Vie parisienne (1866) au cours de laquelle est chanté l'air célèbre du Brésilien. 


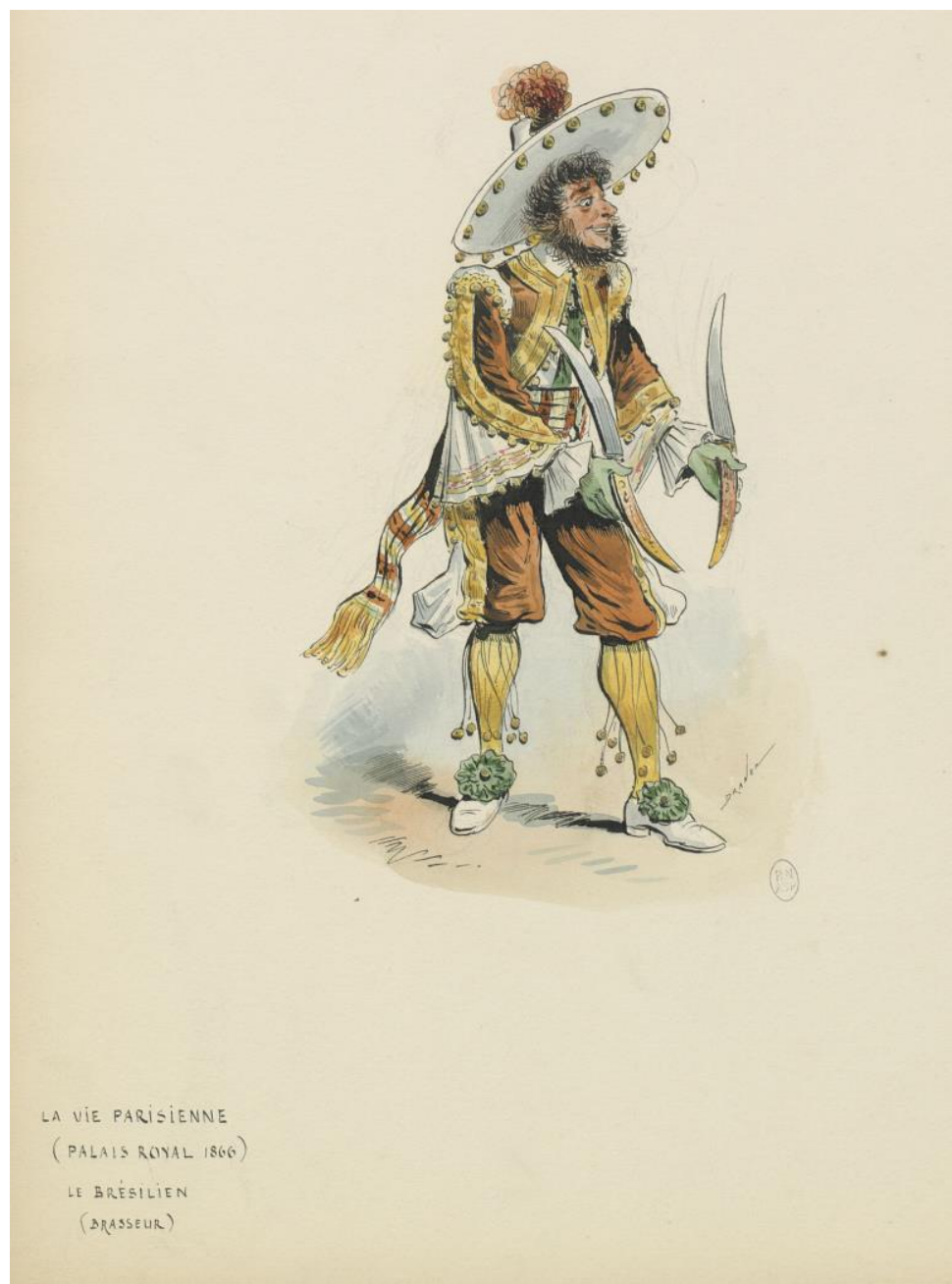

Planche 8. La Vie parisienne. Opéra-bouffe donné le 31 octobre 1866 à Paris. Costume du fameux Brésilien, par Draner. Les deux navajas (couteaux) et le sombrero à pompons sont autant de représentations exotiques mêlant indistinctement des caractères ibériques et latino-américains imaginés pour l'occasion. On les retrouve presqu'à l'identique dans d'autres productions de la même époque. Gallica BNF.

Robinson Crusoe créé par le même prolifique compositeur [Cormon et Crémieux, Paris, 1867] doit être admis avec prudence dans l'inventaire, les références à l'Amérique n'étant que très secondaires (l'île de Robinson se trouve dans les Caraïbes). Signalons cependant les Robinson de Londres [Liney/Sheridan, 1781] et de Naples [Fioravanti /Tottola, 1828].

La piraterie et la flibuste, thèmes ô combien traités en littérature d'aventure, ne semblent pas avoir tenté les librettistes d'opéra. Notre inventaire ne nous permet de distinguer que deux œuvres vraiment consacrées à ce sujet: Die Flibustier [Lobe/Gehe, Weimar, 1829) et Monbar czyli Flibustierowie [Dobrzyński/Paprokki et Pruszakowa, Varsovie, 1863], respectivement consacrées aux redoutables Morgan et Monbar. Cependant, sachons que les pirates apparaissent fréquemment dans les œuvres, comme c'est le cas pour Les Pirates de la Savane [Semet/Bourgeois et Dugué, Paris, 1866], dont le livret accumule les aventures échevelées dans une Amérique latine très colorée (chasse au tigre, héroïne attachée à un cheval fou, etc.).

Jugés plus sérieux, deux thèmes américanistes doivent être cités :

D'une part Atala, qui fera l'objet d'une dizaine de créations dont la grande majorité appartient à la seconde moitié du XIXe siècle : 1802, Paris [Thollé/Chateaubriand]; 1818, Padoue [Pacini/Peracchi] ; 1834, Würzburg [Hörger/Müller] ; 1850, Rome [Sebastiani/Sebastiani] ; 1851, Milan [Butera/Sapio] ; 1876, Milan [Gallignani/Praga] ; 1881, Budapest [Sárosi/Várady] ; 1884, 
Milan [Guglielmi/Cappuccini] ; 1892, Lille [Folville/Colin] ; 1898, Naples [Miceli/Grigico]. Cette distribution tardive est surprenante si on la compare au succès immédiat que le texte de Chateaubriand connut en peinture et en sculpture dès les premières années du siècle.

D'autre part, Manon Lescaut, dont on peut compter sept créations distinctes, pourtant tardives par rapport à la publication originale de Prévost (1731) : Paris, 1820 [Propiac et Gosse] ; Paris, 1830 [Halévy/Scribe et Aumer]; Londres, 1836 [Balfe/Bunn] ; Vienne, 1852 [Strebinger] ; Paris, 1851 [Barrière et Fournier] ; Paris, 1856 [Auber/Scribe] ; Turin, 1893 [Puccini/Illica]. Sachons que les versions de Bellini (1846) et de Massenet (1884) ne peuvent figurer dans notre inventaire : dans la première, Manon est pardonnée et se marie avec Des Grieux sans avoir à quitter la France ; dans la seconde Manon meurt au Havre, sur le bateau devant la conduire en Louisiane.

La fin tragique de Manon nous conduit naturellement en Nouvelle-France, lieu d'intrigues d'une quinzaine d'œuvres entre 1734, Les Mariages du Canada [Lesage, Paris] et 1957, Nouvelle-Orléans [Bastia/Suberville, Paris]. Le thème, peu traité au regard du nombre total des spectacles « américanistes », connaît en somme deux vagues.

Si la première est d'origine exclusivement française, elle ne se développe curieusement qu'après la disparition de la présence française en Amérique du nord et ne sert en fait que de fond à des réflexions philosophiques voire à des intrigues amoureuses : Le Huron [Gretry/Marmontel, Paris, 1768], Candos [Jadin/Delrieu, Paris, 1797], L'ingénu [Piccini, Paris, 1805].

La seconde est en revanche bien plus originale. Etalée sur une vingtaine d'années (1818-1836) et exclusivement italienne, elle s'inspire d'une tragédie déjà ancienne: Hirza ou les Illinois (1767) d'Edme-Louis de Sauvigny, laquelle relate le conflit cornélien déchirant un officier français entre son devoir et son amour pour la fille du chef des Illinois en guerre contre les colons (Fougère, 1994, p. 471-472). Cinq opéras en seront issus : à Milan [Basili, 1818), à Bologne [Sampieri, 1823], à Brescia, [Viviani, 1826], à Trieste [Strepponi, 1829] et à Turin [Coppola, 1836], portant tous le même titre : Gli Illinesi (sauf un : L'Eroe francese, 1826) et du même et prolifique librettiste Felice Romani.

Enfin, n'oublions pas Évangéline [Leroux/Gramont, Bruxelles, 1895], création tardive d'après le poème de Longfellow (1847) consacrée à la déportation des Acadiens.

À la différence de sa rivale française, l'Amérique anglophone bénéficie d'une représentation plus diversifiée tant dans l'origine des productions (anglaises bien entendu, mais aussi françaises, italiennes et même germanique) que dans les thèmes abordés. Une dizaine d'oeuvres peuvent être comptées durant cette période.

Signalons les adaptations des romans de Cooper: The Wept of the Wish-ton-Wish, relatant les rapports entre Indiens et Puritains [Barnard, Londres, 1831 ], The Wigwam [Lee/Brooks, Londres, 1847], bien sûr Les Mohicans [Adam/Guerra, Paris, 1837] et Die Letzen Mohicaner [Genée/Walzel, Munich, 1878]. Signalons également Pocahontas [Solomon/Grundy, Londres, 1884] unique spectacle lyrique consacré à un personnage pourtant célèbre dans le monde anglo-saxon.

Bien curieusement, alors que cette Amérique et ses rapides progrès, et plus encore l'Ouest sauvage attiraient les regards et inspiraient toute une littérature d'aventure (Aimard, Ferry, Le Rouge et bien d'autres); relativement peu de spectacles musicaux et dansés semblent y être consacrés durant cette époque. Outre Le Pays de l'Or déjà signalé, retenons principalement Miss Dollar [Messager/Clairville et Vallin, Paris, 1893] : amours contrariées entre une jeune Américaine et un Français ; Die Dollarprinzessin [Fall/Willner et Grünbaum, Vienne, 1907] relatant les amours de deux riches héritières new-yorkaises ; sans oublier bien entendu la célébrissime Fanciulla del West 
(«La Fille du Far west ) [Puccini/Civinini et Zangarini $]^{35}$. Bien que les personnages du «Cowboy » et de l'Indien redoutable aient été abordés dans des œuvres plus anciennes (Le Pays de l'or notamment), cette romantique et brutale histoire de bandits et de chercheurs d'or peut être considérée comme la première oeuvre lyrique consacrée au «Far-West ${ }^{36}$. La date tardive de cette œuvre doit d'ailleurs être mise en regard du succès rencontré en Europe par les Wild West Shows dont celui de Buffalo Bill fut le plus renommé (1887-1913).

\section{Quatrième époque :}

\section{Le XXe siècle, éclectisme et réflexions}

La Grande guerre n'avait pas seulement enseveli les régiments mais également les certitudes du XIXe siècle et avait donné naissance à un monde instable dont on connaît le destin. Aux bouleversements politiques devaient s'ajouter ceux de la pensée. L'image européenne des Amériques - désormais plus proches par les progrès des communications et des techniques - allait en être profondément affectée, et surtout afficher de multiples visages : arts amérindiens enfin considérés à l'aune de leur valeur ; rythmes nouveaux (jazz, musique tropicale) ; cités tentaculaires et urbanisme vertical ; question raciale et prohibition (au Nord) ; révolutions et pronunciamientos (au Sud). L'art lyrique et chorégraphique en est le fidèle reflet, entrainé par les recherches musicales et les progrès techniques (cinéma, radio).

C'est donc une production scénique certes moins importante (un peu moins de 80 créations) mais bien plus diversifiée et riche qui voit le jour entre la fin de la Première guerre mondiale et nos jours.

Les productions françaises y sont les plus nombreuses : Trente-six de créations dont l'apogée se situe entre 1930 et 1960 ; bien plus nombreuses en cela que les œuvres italiennes (moins de 10) datées principalement des vingt premières années du XXe siècle (et que l'on peut donc considérer comme les ultimes feux du grand mouvement lyrique du siècle précédent). En revanche, la création germanique qui était en retrait depuis le début du XIXe siècle connaît un spectaculaire renouveau (20 œuvres), notamment entre 1980 et nos jours. À moindre échelle, ce renouveau est aussi visible en Espagne ( 7 œuvres). Enfin, sachons que cette époque voit également fleurir des spectacles musicaux issus de lieux jusqu'alors restés en dehors de la production «américaniste »: Lausanne, Prague, Ljubljana, Moscou (?), Amsterdam, Lisbonne. Neuf créations sont ainsi comptées, marques d'un net élargissement de l'image européenne chantée et dansée du Nouveau monde ${ }^{37}$.

$\mathrm{Au}$ sein de la diversité des livrets, et peut-être malgré cela, les thèmes légers et exotiques continuent de connaître un certain succès notamment parmi les productions parisiennes. De la trentaine de créations recensées abordant ce genre, toutes sauf six sont françaises et témoignent ainsi du renouveau de l'opérette parisienne au milieu du XXe siècle et du goût d'un public somme toute assez conservateur ${ }^{38}$. Les œuvres - dont certaines sont devenu célébrissimes - mêlent les situations les plus variées à la caricature souvent outrée des lieux et des personnages : le vérisme étant particulièrement oublié au profit d'un exotisme coloré. Les titres sont à ce sujet fort évocateurs.

\footnotetext{
${ }^{35}$ Voir Zidaric, 2006.

${ }^{36}$ Bien que créée à New-York en 1910, puis à Paris le 16 mai 1912 pour sa première européenne, nous considérerons cette œuvre comme « italienne ».

${ }^{37}$ Notons cependant que, dès les années 1860, des opéras et ballets américanistes avaient vu le jour à Copenhague, Varsovie, Bruxelles, Budapest et Lisbonne.

${ }^{38}$ Pendant les années 1930, 10 des 13 créations « Américanistes » d'Europe sont françaises. Les années 1950 voient ce qui peut être considéré comme l'apogée du gente : les huit opérettes connues sont françaises. 
Signalons les plus connus: La Belle du Far-West [Raynal/Marsan, Paris, 1920] avec sa reconstitution d'un saloon à San Diego et ses danses de « Nègres» californiens (en fait selon les articles de l'époque anciens combattants afro-américains installés à Paris); Toi, c'est moi [Simón/Willemetz et alii, Paris, 1934] et sa complexe intrigue amoureuse d'inversion des rôles sur fond de musique tropicale; La Créole [Offenbach et Hahn/Willemetz et Delance, Paris, 1934], reprise de l'œuvre célèbre d'Offenbach spécialement remaniée pour Joséphine Baker; Au Soleil du Mexique [Yvain et Granville/Mouézy et Willemetz, Paris, 1935], alternant corridas et enlèvement de la belle sur fond de dictature ; Le Chanteur de Mexico [Lopez/Gandéra et Vincy, Paris, 1951], probablement la plus célèbre des opérettes, au cours de laquelle le héros (rôle tenu par Luis Mariano) est séquestré par le faux révolutionnaire Zapata et poursuivi par les assiduités de la volcanique Tornada qui le confond avec son ancien amant ; A la Jamaïque [Lopez/Vincy, Lyon, 1953], lieu du dénouement d'un livret tortueux, ouvert par l'héritage livrant à une charcutière parisienne retraitée une chaine de cinquante snackbars aux Etats-Unis ; Pampanilla [Rys/Nivoix et Hornez, Paris, 1954] dont l'intrigue mêle amours contrariées et coups d'Etat ; enfin Le Corsaire noir [Yvain/Valmy, Marseille, 1958], qui voit le héros à la recherche de sa fiancée (enlevée par un vil ambassadeur espagnol) s'embarquer sur un bateau corsaire dont le second est une femme-pirate déguisée...

L'évolution rapide des mentalités à la fin des années soixante a très certainement joué un rôle non négligeable dans la disparition de ces spectacles exotiques ${ }^{39}$ et l'apparition consécutive d'oeuvres à la fois beaucoup plus sérieuses et «engagées », issues d'horizons plus variés, d'ailleurs souvent germaniques.

Néanmoins, le thème de la Conquête espagnole perdure, ou plutôt renaît après une absence de près de cent ans. Après Montezuma [Sessions/Borgese] donné à Berlin en 1963 (et chanté en plusieurs langues dont le Nahuatl, l'idiome des Aztèques), neuf créations ont en effet vu le jour, toutes consacrées à la chute de l'empire aztèque ${ }^{40}$. Parmi celles-là, signalons La Malinche [Barker, Londres, 1989] et La Noche triste [Prodromides, Nancy, 1989] : premières (et en fait seules) œuvres vraiment consacrées à la fameuse Marina, l'interprète et maitresse de Cortès à qui on accorde un rôle prédominant dans la conquête du Mexique.

C'est depuis les années trente que l'Amérique latine (coloniale et contemporaine) se voit accorder une place au sein des livrets : une dizaine de créations peuvent être comptées depuis Maximilien [Milhaud/Hoffman et Lunel, Paris, 1932], œuvre grave et dénonciatrice tirée du Juarez und Maximilian de Franz Werfel. À l'instar des autres opus de Milhaud comme Bolivar [Paris, 1950], les thèmes en seront variés et d'une valeur historique plus chargée. Citons par exemple : L'étoile et la mort de Joaquin Murrieta [Rybnikov/Grushko, Moscou, 1976] d'après le poème épique de Neruda consacré au bandit-justicier californien ${ }^{41} ;$ Evita [Webster/Rice, Londres, 1978] dédié à Eva Perón ; Viva la vida [Alexiadis/Birke, Frankfort, 1994] relatant la vie de la peintre mexicaine Frida Kahlo ; ou encore Caruso a Cuba [Hamel, Amsterdam, 2019] - dernière œuvre recensée de notre inventaire - consacrée aux angoisses exprimées par le célèbre chanteur après l'attentat à la bombe qui semblait le viser à l'Opéra de Cuba en 1920.

\footnotetext{
${ }^{39}$ Si I'on excepte L'Oncle du Brésil (Bastia/Elzen, Paris, 2000), en fait écrit en 1954 et non joué jusqu'alors.

${ }^{40}$ Desquelles quatre sont germaniques.

${ }^{41}$ Le personnage de Murrieta (ou Murietta) a probablement inspiré Johnston McCulley dans la création de son fameux Zorro. II n'est donc pas surprenant que cette production ait vu le jour en Union soviétique. 


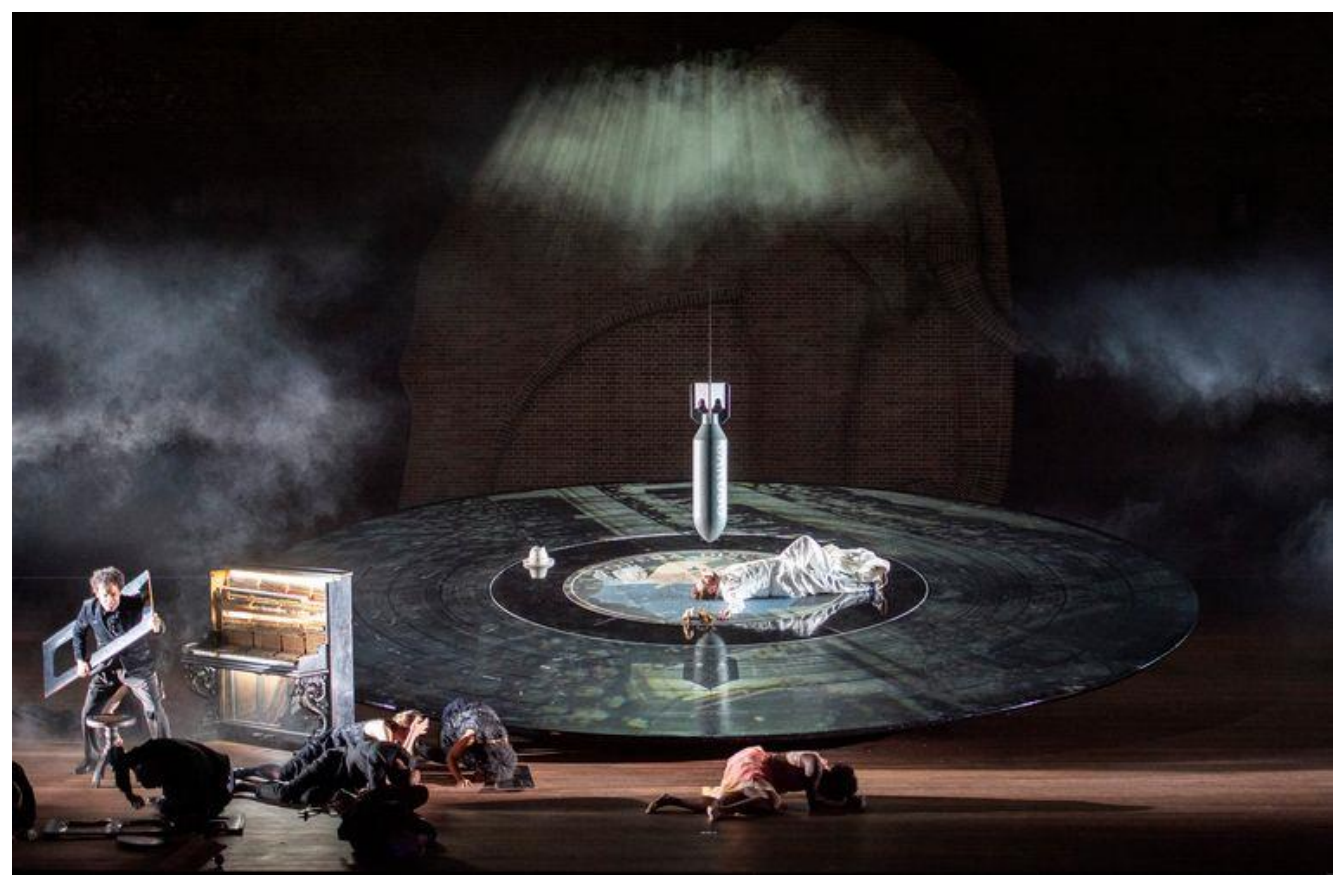

Planche 9. Caruso a Cuba, de Michael Hamel, créé le 3 mars 2019 à Amsterdam. La mise en scène de Johannes Erath et Klaus Berstisch fait appel aux ressources visuelles et sonores les plus contemporaines (musique électronique), bien éloignées de la vision traditionnelle des Amériques. Cliché Monika Ritterhaus in De Verdieping Trouw (4 mars 2019).

Cependant, les créations les plus originales - également les plus récentes - ont pour thème les Etats-Unis ou plutôt les figures marquantes de ce pays. Depuis une quarantaine d'années, une douzaine d'oeuvres peuvent être recensées, sortant des sentiers battus de l'américanisme chanté et dansé : ainsi Charlot [Bacarisse/de la Serna, Madrid] créé en 1933 et consacré au célèbre comédien $^{42}$; Marylin [Ferrero/Bossi, Rome, 1980] dont le livret anglo-italien est consacré à la vie tragique de l'actrice ; The Photographer [Glass, Amsterdam, 1982] dédié à Muybridge, pionnier américain de la photographie; Freeze [Zuidam, Munich, 1994] relatant l'enlèvement de la riche héritière Patricia Hearst ${ }^{43}$; American Lulu [Neuwirth/Strokes, Berlin 2013] dont le livret, inspiré d'Alban Berg, a pour cadre la lutte pour l'égalité des droits civiques dans l'Amérique des années 5070 ; The Perfect American [Glass/Wurlitzer, Madrid, 2013] consacré aux fameux Walt Disney ; ou bien encore Atlas 101 [Mancuso, Trévise, 2013] abordant le rôle de l'actrice Hedy Lamarr et du compositeur Georges Antheil dans l'invention d'un système de cryptage militaire durant la seconde guerre mondiale.

\section{Last but not least : Christophe Colomb, l'amiral chanté et dansé}

Le thème de Christophe Colomb a certainement été le plus traité de toute l'histoire de la représentation des Amériques et s'est traduit depuis plusieurs siècles par une pléthore d'écrits - des plus savants aux plus «inventifs »- sur la vie du personnage ${ }^{44}$. Les représentations en art ont été encore plus nombreuses, quels qu'en fussent les sources et les supports (peintures, fresques, sculptures, monuments commémoratifs) ${ }^{45}$.

\footnotetext{
${ }^{42}$ Signalons également un Chaplin-Ford-Trott [Hartmann/Bormann et Hiller, Munich, 1987] consacré à plusieurs personnalités d'Outre-Altantique.

${ }^{43}$ En 2014, Les Pigeons d'argile [Hurel/Viel] créé à Toulouse abordera de nouveau le sujet.

${ }^{44}$ Voir Mahn-Lot, 1988.

${ }^{45}$ Pas moins de 500 portraits de Colomb sont connus (gravures et peintures). Voir Honour, 1976 ; Pavoni, 1990. 
Cependant, et curieusement, les spectacles traitant de la vie du découvreur - qu'ils fussent théâtraux, musicaux ou autres - semblent avoir été bien moins nombreux. Cette relative discrétion pourrait avoir comme cause les inconnues relatives à la vie du personnage (notamment quant à ses origines), limitant ainsi l'action des librettistes surtout lorsque la dimension nationaliste est engagée $^{46}$.

Les études sur le sujet ${ }^{47}$ nous permettent néanmoins de dénombrer une cinquantaine de spectacles chantés et dansés, consacrés (en partie ou en totalité) au personnage. Sans surprise, les plus nombreux sont italiens (20), une douzaine furent créées en France, dix en Espagne et six en pays germanique ; notons également trois productions anglaises, une tchèque et une portugaise.

La Colombiade chantée et dansée existe depuis plus de trois siècles, distribuée de manière assez irrégulière et dont la forme est bien entendu marquée par les grands courants intellectuels de chaque époque. Bien qu'il ne soit pas impossible que des représentations (ou ne serait-ce que des références) plus anciennes aient existé, Il Colombo ovvero l'India scoperta, donné à Rome en 1690, peut être considéré comme le premier spectacle musical dédié au navigateur ${ }^{48}$. Créée deux siècles après la Découverte, l'oeuvre est évidemment construite à l'aune du Baroque et son livret (du Cardinal Ottoboni) en est le reflet : accompagné de sa femme Anarda, Colomb atteint les Amériques non loin de la «Cittá de Motenzuma» après un voyage long et mouvementé (bataille contre des sirènes). Reçu par Ginacra, empereur du Pérou, il rentre néanmoins en conflit avec ce dernier qui tente de lui ravir Anarda. Mais magnanime, l'empereur la rendra au navigateur (Heck, 1992, p. 243).

Les œuvres suivantes (peu nombreuses : moins d'une dizaine), très certainement inspirées par ce modèle, utiliseront aussi les ressorts du spectacle baroque: exotisme et lointain, mythes antiques et bien sûr grande liberté vis-à-vis des faits historiques. Au sein d'une production essentiellement italienne, notons La Découverte du Nouveau monde, tragédie-lyrique écrite par Rousseau en 1741, et dont le premier acte fut composée par lui-même. Le livret, enrichi par les inévitables intrigues amoureuses, fait apparaître un Colomb généreux et dispensateur de la Paix.

Le début du XIXe siècle, sous l'influence du romantisme naissant, orientera durablement les colombiades (à l'instar de l'ensemble des œuvres américanistes): le découvreur n'est plus le conquérant mais un solitaire doutant de lui, incompris des autres voire méprisé, affrontant l'adversité de la nature et des hommes, et dont le destin tragique s'inscrit parfaitement dans la mode littéraire du temps. Une bonne quinzaine de créations peuvent être comptées durant les deux premiers tiers du siècle, principalement italiennes mais aussi françaises et germaniques.

Cette image perdurera jusqu'à l'extrême fin du siècle et s'inscrira même au sein des cérémonies du quatrième centenaire de la Découverte, matinée il est vrai du vérisme et du naturalisme de l'époque. En 1892 et durant les années suivantes, huit créations verront le jour (dont trois espagnoles). Cristoforo Colombo d'Alberto Franchetti, donné à Gênes, en est l'exemple typique et les trois actes du livret de Luigi Illica en donnent la mesure (Heck 1992, p. 246-249) : à Salamanque - la solitude du héros et les railleries des rivaux; dans les îles - le complot contre sa personne et son retour enchaîné en Espagne ; enfin la vieillesse - les souvenirs et l'ultime volonté de mourir emportant les fers avec lui.

Bien que cette vision hagiographique ne disparaisse pas durant le siècle suivant, elle sera cependant mise à mal par les questionnements que les crises politiques et sociales entretenaient. De

\footnotetext{
${ }^{46}$ À notre connaissance, aucun spectacle chanté et dansé européen ne semble avoir été consacré à d'autres découvreurs, si l'on excepte Vasco Nuñez de Balboa [Chapí/Zapata], zarzuela créée probablement à Madrid en 1872 (Nuñez Ronchi, 2014, p. 500).

${ }^{47}$ Voir Faverzani, 1994 ; Gutiérrez Meza, 2016a, 2016b, 2017 ; Heck, 1992.

${ }^{48}$ Sachons qu'une entrée du Festaufzug de Stuttgart (1599) avait été consacrée à Colomb et à Vespucci, et qu'un des intermèdes des Mettamorfosi d'amore [Mascardi, Gênes, 1623] avait mis en scène l'Amiral. 
la quinzaine d'œuvres comptées entre 1919 et nos jours ( 5 françaises, 4 espagnoles, 3 germaniques, une italienne, une tchèque et une portugaise), plusieurs sortiront des sentiers battus.

Armer Columbus, die Vorgeschichte einer Entdeckung, (Pauvre Colomb, l'histoire antérieure à la découverte) d'Erwin Dressel (Kassel, 1927] en est certainement la première, et dont le livret d'Arthur Zweiniger impose une image caricaturée, voire grotesque du personnage : vision désabusée propre à l'esprit de la République de Weimar (Gutiérrez Meza, 2017, p. 200).

Ce dernier point la distingue du Christophe Colomb de Darius Milhaud, créé en 1930 à Berlin à partir de l'œuvre de Paul Claudel et traduite en allemand par Rudolph Stephan : au soir de sa vie, le navigateur est confronté à ses souvenirs, présentés par un narrateur. L'examen de conscience mais aussi la justification de son œuvre sont affrontés et se mêlent au sein d'une vision associant Histoire et Foi (Faverzani, 1994, p. 23). L'œuvre sera reprise par Jean-Louis Barrault en 1953 puis en 1960, respectivement à Bordeaux puis Paris.

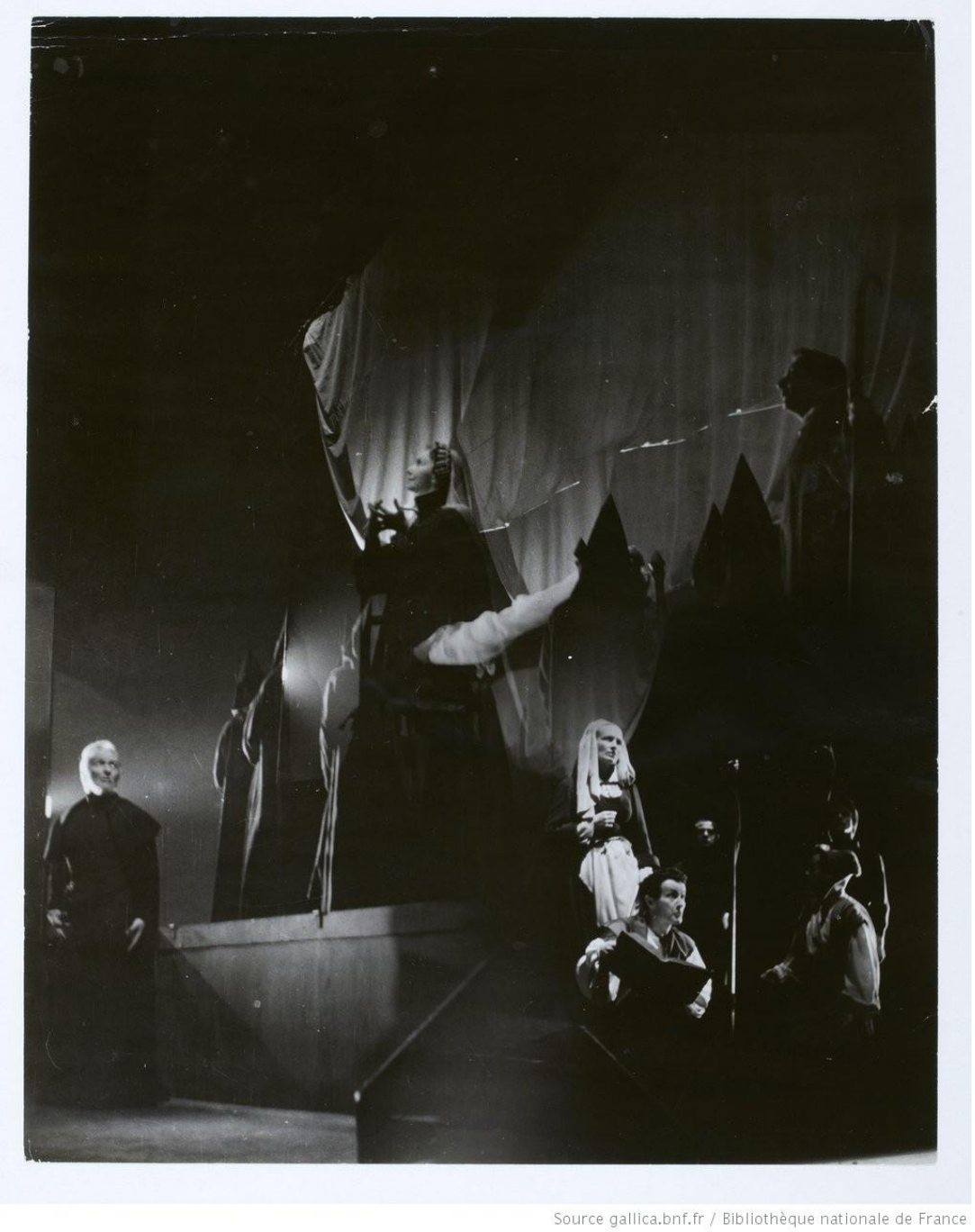

Planche 10. Christophe Colomb de Darius Milhaud et Paul Claudel. Version reprise par Jean-Louis Barrault dès 1953. Cliché 1953 ? : ici Madeleine Renaud dans le rôle d'Isabelle de Castille. Gallica BNF.

Ce concept claudélien est certainement absent de Columbus, Bericht und Bildnis (Colomb, narration et portrait) de Werner Egk, opéra créé à Frankfort en 1942 à partir d'une version radiophonique diffusée en 1933. Le livret (également d'Egk), classique dans sa structure, intègre des éléments empruntés aux principes de Brecht et de Stravinsky, notamment des commentateurs se 
répondant et s'opposant. Ainsi les responsabilités de Colomb sont-elles exposées : dénonciation des abus de la colonisation, échec final de son œuvre ${ }^{49}$.

Signalons également l'Atlántida [1961, Barcelone] curieuse œuvre inachevée de Manuel de Falla, complétée par Ernesto Halffter à partir d'un livret de Jacint Verdaguer, associant le mythe fameux au navigateur.

Néanmoins ne croyons pas que cette vision grave voire pessimiste ait été appliquée à tous les opus de l'univers colombiste. Signalons in fine et pour preuve L'Oeuf-à-voiles ou la véritable découverte de Christophe Colomb, bien curieuse opérette composée en 1937 par Guy Lafarge sur un livret de Cami et Cab, et créée seulement en 1977 à Nantes. Le sujet en est édifiant : Colomb, matelassier pauvre, marié à une mégère, et qui cherche depuis longtemps à faire tenir un œuf debout, entreprend de découvrir l'Amérique à bord d'un bateau-lavoir. La révolte de l'équipage sera évitée grâce à des passagers clandestins particuliers : les «pensionnaires » de Madame Philidor venue fonder une maison accueillante dans le Nouveau monde. Après avoir fui Cuba en proie à une révolution, le groupe arrivera à New York où Madame Philidor ouvrira sa maison et Colomb réussira à faire tenir son œuf...

\section{Conclusion : le spectacle des Amériques, des représentations à la représentation}

Ce rapide survol d'un inventaire n'ayant cessé de grossir au cours de ces derniers mois doit cependant être considéré comme incomplet tant il est certain que bien des références nous ont échappé, et que les œuvres théâtrales (stricto sensu) devront inévitablement y être associées.

Néanmoins, les informations ici rassemblées et qui font suite aux importants travaux précédents nous permettent de dresser une évolution de la vision du continent par la Vieille Europe, depuis maintenant cinq siècles et au prisme d'une forme d'art particulière.

Ainsi pouvons-nous constater que la représentation du Nouveau monde dans son ensemble (peuples présents ou passés, paysages, faune et flore), découvert et identifié lentement au sein de spectacles, a été avant tout le reflet de notre monde : un Autre et un Ailleurs imaginé, inventé à l'aune des considérations politiques, religieuses, philosophiques et esthétiques des élites européennes, principalement de France et d'Italie.

Cependant, bien loin d'y considérer une forme d'ethnocentrisme borné nous devons y voir un échange : l'intégration réciproque d'un continent à l'autre, métissage, syncrétisme, création d'un espace hybride chère à la pensée de Gruzinsky (1999); en somme enrichissement non seulement intellectuel mais aussi artistique.

Car ces œuvres du spectacle «américaniste », malgré les grandes différences qu'elle peuvent afficher, tant dans le contenu des « arguments » et des livrets que par leur nature (parade, art lyrique, danse), ont un point commun primordial, condition sine qua non de leur existence : le spectacle et son double corollaire - la scène qu'il faut occuper, et le public que l'on doit conquérir. Les conséquences en sont plus que visibles: décors, machineries, costumes et accessoires. Tous ces éléments, jouant un rôle essentiel dans cette vaste gamme de productions, participent à la représentation visuelle et «théâtrale » (au sens large du terme) d'un monde exotique et lointain.

Cette dernière, comme nous l'avons vu, est per natura soumise aux connaissances du moment, aux modes, aux époques et aux lieux de production ou bien encore aux conditions culturelles et politiques dans lesquelles elles apparaissent.

\footnotetext{
${ }^{49}$ Bien que ces thèmes abordés eussent pu être condamnés par le régime nazi, et malgré les critiques, l'oeuvre ne fut pas interdite. Voir Gutiérrez Meza, 2016b. 
Bien des exemples iconographiques nous sont parvenus et sont d'un grand intérêt ; tels ceux présentés ici. L'étude de ces documents, que nous n'avons pu considérer dans le cadre de cet essai, doit donc être menée et sera la prochaine étape de notre travail.

\section{Bibliographie}

\section{Monographies}

d'Aglie' Filippo, Il Tabacco. Balletto,Torino, 1650, Biblioteca Nazionale Universitaria, Torino. [https://www.internetculturale.it/]

d'Aglie' Filippo, L'Unione per la peregrina Margherita Reale e Celeste, 1560, Biblioteca Nazionale Universitaria, Torino. [https://www.internetculturale.it/]

Almaraz Arroyo Antonio, «América como texto y como pretexto en El Artista », Arbor Ciencia, Presencia y Cultura, vol.188-757, 2012 (p. 947-57).

Apollonio Silvia, «Appunti su Le Mettamorfosi d'amore (1623) di Agostino Mascardi », in G. Baldassarri et alii (a cura di), La letteratura degli italiani 4. I letterati e la scena, Adi editore, Roma, 2014 (p. 1-7).

Arenas Frutos Isabel, « Aspectos culturales de la historiografía cortesiana en España (1940-1989) », Revista de Indias, vol. L-188, 1990, Madrid, (p. 277-288).

Asseline David, Antiquitez et Chroniques de la ville de Dieppe [1682], avec une introduction et des notes historiques par MM Michel Hardy, Guérillon et l'Abbé Sauvage (deux vol.), Marais, Dieppe, 1874.

Assereto Giovanni, Isengard, Luigi d', Dizionario Biografico degli Italiani, Volume 62, 2004. Treccani (Istituto Giovanni Treccani). [https://www.treccani.it/enciclopedia/]

Auzolle Cécile, «Les créations lyriques à l'Opéra de Paris entre 1945 et 1955 », in Noiray Michel et Solveig Serre, Le répertoire de l'Opéra de Paris (1671-2009), École nationale des Chartes, Paris, 2010 (p. 103-113).

Bernabéu Albert Salvador, «La Conquista después del desastre. Guatimozín y Hernán Cortés. Diálogo (1899), de Francisco Pi y Margall », Estudios de Historia Novohispana, $n^{\circ} 21,1999$ (p. 107-144).

Billington Michael, «Evita review - breathtaking inventiveness and quicksilver fluency », The Guardian International Edition, 22 sept 2014. [https://www.theguardian.com/stage/2014/sep/22/evita-review-andrew-lloyd-webber-theatre]

Boorsch Suzanne, «America in Festival Presentations », in Chiappelli et alii, First images of America, University of California Press, Berkeley, 1976 (p. 503-515).

Bujok Elke, « Der aufzug der Königin Amerika in Stuttgart », Tribus 52, Linden-Museum, Stuttgart, 2003 (p. 80-110).

Cahingt Henri, De Dieppe en 1527. Lettres d'un voyageur, Imp. Bertout, Luneray, 1980.

Calhoun Winton, John Gay and the London Theatre, University Press of Kentucky, Lexington, 1993.

Castillo Moisés R., Indios en escena: la representación del amerindio en el teatro del Siglo del Oro, Purdue University Press, West Lafayette, 2009.

Chinard, Albert, L'Amérique et le rêve exotique dans la littérature française au XVIIe et XVIIIe siècles, Paris, Hachette, 1913.

Cirino Andrea, Feste celebrate in Napoli per la nacita del Serenissimo prencipe di Spagna, Faggioli, Naples, 1658.

Clément Félix et Pierre Larousse, Dictionnaire des Opéras, Grand dictionnaire Universel, Larousse, Paris, 1881.

Comas José, «Una ópera sobre 'El otoño del patriarca', de García Márquez, triunfa en Bremen », El País, Madrid, 9 de junio, 2004. [https://elpais.com/diario/]

Corder F., « The works of Sir Henry Bishop », Musical Quarterly, Vol. 4, Oxford, Janv 1918, (p. 78-97).

Denis Ferdinand, Une fête brésilienne célébrée à Rouen en 1550, Techener, Paris, 1850.

Dratwicki Alexandre, "Gossec et la danse ou les premiers pas du ballet-pantomime français. Autour du succès de Mirza », in Dratwicki et alii (coord.) François-Joseph Gossec (1734-1829), Centre de Musique baroque de Versailles, Versailles, 2002 (p.101-116). 
Duviols Jean-Paul, «Montezuma, Opéra en trois actes, musique de Karl Friedrich Graun, livret de Frédéric II, roi de Prusse », In Lucien Clare, Jean-Paul Duviols, Annie Molinié (coord.), Fêtes et divertissements, Presses de l'Université de Paris-Sorbonne, Paris, 1997 (p. 221-225).

Faverzani Camillo, «Christophe Colomb, héros d'opéra en France et en Italie entre XIXe et Xxe siècles », in Jacques Houriez (coord.), Christophe Colomb et la découverte de l'Amérique: mythe et histoire (actes du colloque international organisé par l'Université de Franche-Comté, les 21-23 mai 1992), Presses de l'Université de FrancheComté, Besançon, 1994 (p. 17-34).

Feest Christian, "The People of Calicut : objects, texts and images in the age of Proto-Ethnography », Boletim do Museum Paraense Emilio Goeldi. Ciencas Humanas, 9-2, Belém, 2014 (p. 287-303).

Fétis François-Joseph, Biographie universelle des musiciens (10 vol.), Firmin-Didot, Paris, 1883.

Fifield Christopher, «Michael BALFE (1808-1870) The Maid of Artois » in MusicWeb, Recording of the month, Feb. 2005. [http://www.musicweb-international.com/classRev/2005/Dec05/Balfe_2042-3.htm].

Fougère Éric, «Billardon de Sauvigny et l'innocence perdue du premier âge », Dix-huitième Siècle, n²6, Paris, 1994 (p. 465-474).

Freeman Daniel E., Josef Mysliveček, "Il Boemo" : The man and his Music, Sterling Heights, Harmonie Park Press, 2009.

Frohock Richard, "Sir William Davenant's American Operas», The Modern Language Review, Vol. 96-2, Cambridge, 2001 (p. 323-333).

Garavaglia Andrea, Il mito delle Amazzoni nell'opera barocca italiana, Università degli Studi di Milano, Milan, 2015.

Ghilione Marco, «Nuovo mondo in ... cantato. Le musiche dedicate all'impressa di Cristoforo Colombo », New Magazine Imperia, $\mathrm{n}^{\circ}$ 4, Agosto 2014 (p. 45-55).

Girardi Michele, «Appunti su Alzira», in Alzira, [Programme de la Saison Verdi 1990-91], Teatro Regio, 1990, Parme (p. 5-27).

Girardi Michele, «Un Felice Motezuma » Conférence prononcée lors du congrès international Columbus, Montezuma und Cortés als Bühnengestalten. Zur Rezeption-sgeschichte der Entdeckung Amerikas, Villa Vigoni (Como), 4-6 maggio 1993. [http://www-5.unipv.it]

Grammeniati Barbara, «Filippo d'Agliè (1604-1667) Ballets and Musical Style », in Grammeniati B. (edit), Seventeenth-Century Ballet A multi-arts spectacle, Xlibris Corporation, Bollmington, 2011 (p. 79-101).

Griffel Margaret Ross, Operas in English. A dictionary (Revised edition), Rowman and Littlefield, Lamham, 2013.

Griffel Margaret Ross, Operas in German. A dictionary (Revised edition), Rowman and Littlefield, Lamham, 2018.

Gruzinsky Serge, La pensée métisse, Fayard, Paris, 1999.

Guénin Eugène, Ango et ses pilotes, Prudhomme, Paris, 1901.

Gutiérrez Meza José Elías, 2016a, «Secretos colombinos: Cristóbal Colón de Leonardo Balada y Antonio Gala », Iberoamericana, VI-63, Berlin, 2016 (p. 205-224).

Gutiérrez Meza José Elías, 2016b, «Columbus de Werner Egk: una ópera sobre Cristóbal Colón en la Alemania nazi », Revista de Filología Románica, 33, Madrid, 2016 (p. 89-97).

Gutiérrez Meza José Elías, «La imagen de Cristóbal Colón y el Descubrimiento de América en la ópera alemana durante el Tercer Reich », Memoria y Civilización, 20, Pamplona, 2017 (p. 199-213).

Heck Thomas, «The operatic Christoper Columbus : three hundred years of Musicac Mythology », in Mancini Albert and Dino Cervigny (edit), Images od America and Columbus in Italian Literature, Annali d'Italianistica, v. 10, Chapel Hill, 1992 (p. 236-273).

Heine Christiane, «Salvador Bacarisse (1898-1963) en el centenario de su nacimiento », Cuadernos de Música Iberoamericana, 5, Madrid, 1998 (p. 43-74).

Highfill Philip H. Jr., Kalman A. Burnim and Edward A. Langhans, Biographical Dictionary of Actors, actresses, musicians, dancers, managers \& other stage personal in London. 1660-1800, Southern Illinois University Press, Carbondale, 1973.

Holmstead Andrea, Roger Sessions, a Biography, Routledge, New-York-London, 2008.

Honour Hugh, The New Golden Land. European Images of America, Allen Lane, London, 1975. 
Honour Hugh, «L'image de Christophe Colomb », Revue du Louvre, 26, Paris, 1976 (p. 255-267).

Hoskins Robert (Edit), The Pantomime of Robinson Crusoe (1781), Artaria Editions, London, 2005. [https://issuu.com/artaria-editions/docs/ae447_c]

Ilustración Artística (La), n 524, Barcelona, 1892.

Kučera Martin, La mise en scène des pièces de Paul Claudel en France et dans le monde (1912-2012), Olomouc, 2013. [https://docplayer.fr/]

Kunz Isabel, Inkle und Yariko. Der Edle Wilde auf den deutschsprachigen Bühnen des ausgehenden 18. Jahrhunderts, Inaugural-Dissertation zur Erlangung des Doktorgrades der Philosophie an der Ludwig-Maximilians-Universität, München, 2007. [https://edoc.ub.uni-muenchen.de/6921/]

Lacroix Laurier, «Quand les Français jouaient aux Sauvages... ou le Carrousel de 1662 », Annales d'Histoire de l'Art Canadien, vol 3-1/2, Québec, 1976 (p. 44-54).

Letellier Robert Ignatius, Daniel-François-Esprit Auber: The Man and His Music, Cambridge Scholars Publishing, Newcastle, 2010.

Letellier Robert Ignatius, Operetta: A Sourcebook, Volume II, Cambridge Scholars Publishing, Newcastle, 2015.

Liebermann Rolf (direct.), Dictionnaire chronologique de l'Opéra, Ramsay, Paris, 1994.

Lischke André, Guide de l'opéra russe, Fayard, Paris, 2017.

Lonchampt Jacques, «" Amerika ", d'Haubenstock-Ramati d'après Kafka », Le Monde, 20 octobre 1966.

MacDougall Hugh, Introduction to the The Wept of the Wish-Ton-Wish, James Fenimore Cooper Society Website, 2009. [https://jfcoopersociety.org/DRAMA/wept.html]

Malandrain Thierry, «Victor Natta», Malandrain Ballet Biarritz, $\mathrm{n}^{\circ} 88$ (Journal d'information du Centre chorégraphique national de Nouvelle-Aquitaine), 2001 (p. 8-30).

Marcello Elena, Girolamo Gigli. Un pazzo guarisce l'altro, Biblioteca Pregoldoniana, Lineadacqua Edizioni, Venezia, 2016.

Martini Dario Guglielmo, Bibliografia Colombiana Teatrale, Comitato Nazionale Cristoforo Colombo, 2011. [www.cristoforocolombo.com]

McGowan Margaret M., « Deux fêtes en Savoie en 1644 et 1645 », Baroque, 5, Montauban, 1972, (p. 49-58). [https://journals.openedition.org/baroque/373]

Mahn-Lot Marianne, Portrait historique de Christophe Colomb, Paris, Seuil, 1988.

Mongne Pascal, «Les collections françaises d'objets ethnographiques, d'art colonial et d'art populaire originaires de l'aire de la Nouvelle-France. » (avec Marie-Bénédicte Seynhaeve-Kermorgant), in Mémoires Amérique française, Portail Internet de la Commission Franco-Québécoise sur les Lieux de Mémoire Communs (avec la collaboration de Emilie Bel, Pauline Cailleux, Marine Pichard et Thibaut Vergé), 2013. [http://www.memoiresameriquefrancaise.com/]

Mongne Pascal, «L'indien "emplumé" ou la caricature d'un continent», in Fabien Ferrer-Jolly (coord.), Plumes. Vision de l'Amérique précolombienne, Exposition tenue au Musée du Quai Branly, Paris (2016-2017), Musée des Jacobins d'Auch - Somogy, Paris, 2016 (p. 106-117).

Mongne Pascal, 2018 ${ }^{\mathrm{a}}$ "Las colecciones de arte mexicano en los museos de Francia : historia y patrimonio comunes », in Hernán Salas Quintanal, Mari Carmen Serra Puche y Alberto Vital (coord.), El Patrimonomio : Dialogo cultural entre México y Francia (Coloquio Internacional del Centro de Estudios Mexicanos de la Unam en Francia, 1 et 2 juin 2015), UNAM, México, 2018 (p.101-140).

Mongne Pascal, 2018b L'image de la Nouvelle-France dans les arts de France, Exposition virtuelle au sein de la Commission Franco-Québécoise sur les Lieux de Mémoire Communs. [https://www.cfqlmc.org/]

Mongne Pascal, «Le reflet du Vrai: répliques, pastiches et faux en art précolombien », in Inés Mónica SarmientoAcher and Leonor Taiano Campoverde (édit.), The second World Congress of Universities and researchers (22-24 August 2019), Saint-John's University, New York, 2019 (p. 53-66).

Moureau François, «Les Amérindiens dans les ballets de cour à l'époque de Champlain », in Litalien Raymonde et Denis Vaugeois (édit.), Champlain, la naissance de l'Amérique française, Septentrion, Québec, 2004 (p. 43-49).

Moureau François, «Danses amérindiennes à la cour », in François Moureau (dir.), Le Théâtre des voyages. Une scénographie de l'Âge classique, PUPS, Paris, 2005 (p. 1-18). [http://cour-de-france.fr/article1331.html] 
Núñez Ronchi Ana, "Los americanos en las escenas líricas europeas : de los hermanos Purcell (1695) a Carl Heinrich Graun (1755) », Revista de Indias, vol.74-261, CSIC, Madrid, 2014 (p. 483-506).

Onesti Stefania, Dietro la traccia de «Gran Maestri » prassi e poetica del ballo pantomimo italiano negli ultimi quarant'anni del settecento, Thèse doctorale, Universita degli Studi di Padova, 2014. [http://paduaresearch.cab.unipd.it/6332/].

Paquot Marcel, "Les étrangers dans le ballet de cour au temps de Henri IV », Revue du Seizième siècle, T. 16, 1929, Paris, (p. 21-39).

Pavoni Rosanna, Christophe Colomb : images d'un inconnu, Vilo, Paris, 1990.

Perrone Moisés Beatriz, «L'Alliance normando-tupi au XVIe siècle : la célébration de Rouen », Journal de la Société des Américanistes, 94-1, 2008, Paris (p. 45-64).

Pisani Michael, Imagining Native America in Music, Yale University Press, New Haven, 2005.

Pisani Michael, A Chronological Listing of Musical Works on American indian Subjects Composed since 1608, Vassar College, 2006. [http://indianmusiclist.vassar.edu/]

Polzonetti Pier Paolo, Italian Opera in the Age of the American Revolution, Cambridge University Press, Cambridge, 2011.

Ponzetto Valentina, «Henri de Saint-Georges, Hippolyte Monpou, Le Planteur, opéra-comique en deux actes », Studi Francesi, 179 (LX-II), Turin, 2016, p. 345.

Quignard Brigitte, «Document: la fête cannibale de 1550 », in Jean-Claude Arnould et Emmanuel Faye (coord.), Rouen 1662. Montaigne et les Cannibales, Actes du colloque organisé à l'Université de Rouen en octobre 2012, Publications numériques du CérÉdI, Actes du Colloque $\mathrm{n}^{\circ}$ 8, Rouen, 2013. [http://ceredi.labos.univrouen.fr/public/?document-la-fete-cannibale-de-1550.html]

Ragazzi Franco, «Teatri e scene nell'età del Liberty in Liguria», Docplayer Italie, s.d., (p. 33-39). [https://docplayer.it/1800278-Teatri-e-scene-nell-eta-del-liberty-in-liguria.html]

Rainer Werner, «Aus dem Vorwort zur Partitur von P. Florian Reichssiegel », [introduction au programme], in Der Traum, MH 84 Eine Pantomime in zwei Aufzügen..., Mozart-Opern Institutes, Salzburg, 25-27 April 2012. Revue de Paris, Tome Seizième, Paris 1835.

Rice John A., Salieri and Viennese Opera, University of Chicago Press, Chicago, 1998.

Saby Pierre, "Cataclysme et exotisme dans l'opéra français : Les Incas du Pérou (Rameau, 1735) et Cora (Méhul, 1791) », in Anne-Marie Mercier-Faivre et Chantal Thomas (coord.). L'invention de la catastrophe au XVIIIe siècle. Du châtiment divin au désastre naturel, Droz, Genève, 2008 (p.419 - 431).

Samoggia Luigi, «Dardani Paolo», in Dizionario Biografico degli Italiani (Volume 32 (1986), Treccani (Istituto Giovanni Treccani).[https://www.treccani.it/enciclopedia/]

Sanchez Jean-Pierre, «Voltaire et sa tragédie américaine Alzire (1736)», Caravelle n58, L'image de l'Amérique latine en France depuis cinq cents ans, Toulouse, 1992 (p. 17-37).

Savage Roger, «Rameau's American Dancers », Early Music, Vol. 11-4, Rameau Tercentenary Issue, Oxford, 1983 (p. 441-452).

Schmidt Carl, «Antonio Cesti's "Il pomo d'oro": A Reexamination of a Famous Hapsburg Court Spectacle », Journal of the American Musicological Society, Vol. 29-3, Autumn 1976 (p. 381-412).

Schwarz Max, Johann Christian Bach (1735-1782), in Fleischer Oskar und Johannes Wolf, Sammelbände der Internationalen Musikgesellschaft (2 Jahrg., H. 3), Druck und Verlag von Breitkopf und Härtel, Leipzig, 1901 (p. 401-452).

Siglo Pintoresco, Tomo 2, Madrid ,1946. [https://archive.org/details/elsiglopintoresc02]

Thomazi Auguste, Les flottes d'or, Payot, Paris, 1956.

Tkaczyk Viktoria, «L'École d'ingénierie scénique de Giulio Parigi (1608-1680). Giulio Parigi’s "School of Theater Engineering”, 1608-1680 », Artefact, Techniques, histoire et sciences humaines 4, Paris, 2016 (p. 99-117).

Tobias Tobi, «Home Truths», The New York Times Magazine, Oct. 24, 1983 (p. 115). [https://books.google.fr/books?id=l-ICAAAAMBAJ\&q=rob+coe+the + photographer + muybridge] 
Tomasevic Nika, «Benezor and La morte di Pizarro: portrayal of Italo-French political and cultural confrontation in Rome at the end of the Eighteenth century », in Arianna Fabbricatore, La Danse et les Nations [programme détaillé], Colloque des 26-18 octobre 2017, Paris. [https://hddanse.hypotheses.org/233]

Van Bellen Eise Barel, Les Origines du mélodrame, Keming \& Zoon, Utrecht, 1927.

Vazzoler Franco, «Don Chisciotte e le "genti americane". Comicita ed esotismo nell'Atalipa, dramma per musica di Gerolamo Gigli », in Mancini Albert and Dino Cervigny (edit), Images od America and Columbus in Italian Literature, Annali d'Italianistica, v.10, Chapel Hill, 1992 (p190-212).

Viale Ferrero Mercedes, Histoire de l'Opéra italien, Volume 5 : l'Opéra spectacle, Mardaga Editeur, Liège, 1992.

Vila Marie-Christine, Quatre siècles d'opéra : 140 oeuvres lyriques de la Renaissance à nos jours, Larousse, Paris, 2002.

Wehner Ralf, «Columbus in der Musik des 19. Jahrunderts », in Michael Zeuske, Bernd Schröter und Jörg Ludwig (eds.), Sachsen und Lateinamerika. Begegnungen in vier Jahrhunderten, Vervuert, Frankfurt am Main, 1995 (p. 267-278).

Worrall David, Harlequin Empire, Race, Ethnicity and the Drama of the Popular Enlightenment, Pickering \& Chatto, Londres, 2014.

Wild Nicole, Décors et costumes du XIXe siècle. Tome I : Opéra de Paris, Éditions de la Bibliothèque nationale de France, 1987.

Wild Nicole, Décors et costumes du XIXe siècle. Tome II : Théâtre et décorateurs, Éditions de la Bibliothèque nationale de France, 1993.

Wild Nicole et David Charlton, Théâtre de l'Opéra-Comique Paris: répertoire 1762-1972, Mardaga. Bruxelles, 2005.

Zavala Huguette, América inventada : fiestas y espectáculos en la Europa de los siglos XVI al XX, Banco de Santander, Madrid, 1994.

Zidaric Walter, «De David Belasco à Giacomo Puccini : La Fanciulla del West, premier opéra "américain" », Revue LISA/LISA e-journal, Vol. IV - ${ }^{\circ} 2$ : Opera and National Identity in the English-speaking world, 2006 (p. 52-67). [https://journals.openedition.org/lisa/2106]

Zieziula Grzegorz, «Le Flibustier emprisonné dans une "pièce à sauvetage": Monbar, héros de l'opéra de Dobrzyński », Théatre et Drame Musical, 4 (7-8), 2006, (p. 151-162).

\section{Bases de données en ligne}

Adelphi Theatre Project (the), [https://www.umass.edu/AdelphiTheatreCalendar/]

Alte Oper (die), [https://www.alteoper.de/de/programm/]

Art Lyrique Français, Encyclopédie de l'art lyrique français, [https://www.artlyriquefr.fr/index.html].

Balletopera, Ballets créés à l'Opéra de 1832 à 1899, [http://balletopera19eme.canalblog.com/archives/]

BNF Data, [https://data.bnf.fr]

British Theatre Guide, [https://www.britishtheatreguide.info/]

CESAR, Calendrier électronique des spectacles sous l'Ancien régime et la Révolution, [https://cesar.humanum.fr/cesar2/]

Corago, Alma Mater Studiorum, Universitá di Bologna, [http://corago.unibo.it/]

Curiosity Collections Harvard Library, [https://curiosity.lib.harvard.edu/]

Dezede, Archives et chronologie des spectacles, [https://dezede.org/]

Deutsche Digital Bibliothek, [https://www.deutsche-digitale-bibliothek.de/]

ECMF, Encyclopédie multimédia de la comédie musicale théâtrale en France 1918-1944, [https://comediemusicale.jgana.fr/]

Fondazione Giorgio Cini, [https://www.cini.it/]

Gallica, [https://gallica.bnf.fr/accueil/fr/]

Gaspari, Catalogo de la Biblioteca di Musica di Bologna, [www.bibliotecamusica.it] 
Gilbert and Sullivan Archive, [https://gsarchive.net/]

Hathitrust Digital Library, [https://catalog.hathitrust.org/]

Internet archive, [https://archive.org/]

Internet Culturale, Cataloghi ecollezioni delle biblioteche italiane, [https://www.internetculturale.it/].

Iopera.es, [https://iopera.es/]

ItalianOpera, La ricerca musicale in Italia, [https://www.italianopera.org/].

IRCAM Ressources, Centre Pompidou, [https://www.ircam.fr/ressources/]

Kulturportal West-Ost, [https://kulturportal-west-ost.eu/biographien]

Library of Congres, [https://www.loc.gov]

Libretti d'Opera italiani, [www.librettidopera.it]

Librinlinea, Biblioteche piemontesi on line, [http://www.librinlinea.it/]

Llista de personatges històrics d'òpera, [https://ca.wikipedia.org/wiki/]

Maestro Francisco Alonso, [https://www.maestroalonso.com/todas-las-obras/]

MDZ, Münchener DigitalisierungsZentrum Digitale Bibliotek, [https://www.digitale-sammlungen.de/en/]

Münchener Biennale, [http://archive.muenchener-biennale.de/archiv/]

New York Public Library, [https://www.nypl.org/research/research-catalog/]

Nic Muni, [http://www.nicmuni.com/]

OPAC SBN, Catalogo del Servizio Bibliotecario Nazionale, [https://opac.sbn.it/opacsbn/opac/iccu/free.jsp]

OPAC Sicilia, [http://opac.sicilia.metavista.it/]

Oper in Italien und Deutschland zwischen 1770 und 1830, [http://www.oper-um-1800.uni-mainz.de]

Opera Archief NL, [https://opera-archief.nl/index.php/]

Opera Baroque, Le Magazine de l'opéra baroque, [https://operabaroque.fr]

OperaPoint, [http://www.operapoint.com/?p=1506]

Opera Roma, Archivio Storico, [https://archiviostorico.operaroma.it/]

Opérette - Théâtre Musical, [https://www.operette-theatremusical.fr]

Operetta research Center, [http://operetta-research-center.org/]

Operetten-Lexikon, [https://www.operetten-lexikon.info/]

Philidor, Portail de Ressources du pôle de recherche du Centre de musique baroque de Versailles, [http://philidor.cmbv.fr]

Real Biblioteca,Patrimonio Nacional, [https://realbiblioteca.patrimonionacional.es]

ResMusica, [www.resmusica.com/]

RISM, Répertoire international des sources musicales, [https://opac.rism.info/]

Société Paul Claudel, [https://societe.paul-claudel.net/misesenscene/]

Stanford University Libraries, «Opening Night, Opera and oratorio premieres », [https://exhibits.stanford.edu/operadata]

Teatro de la Abadia, [https://www.teatroabadia.com/es/]

Teatro La Fenice, Archivio Storico, [http://archiviostorico.teatrolafenice.it/]

Teatro.it, [https://www.teatro.it/recensioni/]

Théâtre Classique, [https://www.theatre-classique.fr/pages/programmes/edition.php?t=../documents/]

Théâtre-Documentation, [http://théâtre-documentation.com/]

Théâtre Français de la Révolution à l'empire, [http://theatre1789-1815.e-monsite.com/]

Theatermuseum, Wien, [https://www.theatermuseum.at/it/onlinesammlung/] 
TUB, Technische Universität Braunschweig, [https://katalog.ub.tu-braunschweig.de/vufind/] Vanderbilt University Heard Library, [https://catalog.library.vanderbilt.edu/discovery/] Warwick Digital Collections, University of Warwick, [https://cdm21047.contentdm.oclc.org/] Wayback Machine, [https://web.archive.org/web/] 